\title{
Concurrent optimization of process parameters and product design variables for near net shape manufacturing processes
}

\author{
Daniele Marini $^{1}$ (D . Jonathan R. Corney ${ }^{1}$
}

Received: 12 April 2019 / Accepted: 20 May 2020 / Published online: 12 June 2020

(c) The Author(s) 2020

\begin{abstract}
This paper presents a new systematic approach to the optimization of both design and manufacturing variables across a multistep production process. The approach assumes a generic manufacturing process in which an initial near net shape (NNS) process is followed by a limited number of finishing operations. In this context the optimisation problem becomes a multivariable problem in which the aim is to optimize by minimizing cost (or time) and improving technological performances (e.g. turning force). To enable such computation a methodology, named conditional design optimization (CoDeO) is proposed which allows the modelling and simultaneous optimization of process parameters and product design (geometric variables), using single or multi-criteria optimization strategies. After investigation of CoDeO's requirements, evolutionary algorithms, in particular Genetic Algorithms, are identified as the most suitable for overall NNS manufacturing chain optimization The $\mathrm{CoDeO}$ methodology is tested using an industrial case study that details a process chain composed of casting and machining processes. For the specific case study presented the optimized process resulted in cost savings of $22 \%$ (corresponding to equivalent machining time savings) and a $10 \%$ component weight reduction.
\end{abstract}

Keywords Manufacturing optimization · Process optimization · Design optimization · Near net shape $\cdot$ Genetic algorithm Machining parameters optimization

\section{Introduction}

The global desire to reduce energy, material consumption and emissions continuously push companies to adopt manufacturing technologies that reduce wastages and maintain productivity. In response to these drivers near net shape (NNS) manufacturing processes have emerged as a powerful tool for achieving such savings.

NNS manufacturing is a relative, rather than absolute, propriety of a process chain that minimizes raw material utilization and finishing machining operations (Altan and Allen 1990) in comparison with the alternatives (Marini et al. 2017a).

Optimization of such processes must take a broad view and control both process parameters and design variables in order to minimize the raw material utilization and the time

Daniele Marini

daniele.marini@strath.ac.uk; daniele.marini19@gmail.com

1 Department of Design, Manufacturing and Engineering

Management (DMEM), University of Strathclyde, 75

Montrose St, Glasgow G1 1XJ, UK of machining operations. The determination of optimal, or acceptable near-optimal, solutions by a suitable optimization technique has been recognized as critical but difficult task for researchers and practitioners (Mukherjee and Ray 2006).

However, in industrial enterprises and academic literature, product design and manufacturing process optimization are linked by predominately qualitative " Design-for-X" methodologies rather than the explicit setting of dimensions and process variables. In contrast, this paper presents a "offline" methodology for selecting the best configuration of design and process parameters in a NNS supply chain, which integrates both product and process design.

\section{Aim and objectives}

The main aim of the NNS optimization approach proposed here is to minimize cost (or time) and improve technological performances (e.g. minimizing turning force) through systematic variation of product design and manufacturing process parameters.

The aim gives rise to two objectives: 
1. Define an optimization methodology

2. Quantify benefits by implement it on a case study

The proposed methodology, known as conditional design optimization (CoDeO), is limited to a NNS manufacturing chain: where, a NNS chain is composed of a primary shaping process and a limited number of machining steps.

\section{Methodology}

In other contexts the idea of concurrent optimization between process parameters, manufacturing systems and design have been explored using different methodologies.

In mechatronic, quantitative approaches have been adopted for developing "Design for Control" methodologies for concurrent optimization. Authors, such as Bi and Zhang (2001), Li et al. (2001), Rampersad (1995) and Ouyang et al. (2004), develop concurrent mathematical models able to control robot mechanics and concurrently optimize robot dynamics and product design. In comparison to these approaches, an "online" (i.e. real-time) process and product optimization of flexible systems is beyond the scope of this paper.

Elsewhere concurrent optimization methods have been extended to applications in operations management and management science. Qualitative and quantitative methodologies, such as Zhang et al. (2019) and Ha and Porteus (1995), have been developed for modelling the links between manufacturing and design operations, allowing their optimal scheduling and design. However, such a systemic and holistic managerial view of the enterprise systems (Zhang and Wang 2016; Giachetti 1998) is beyond of the scope of this work.

In the context of NNS manufacturing, the machining steps gives an opportunity to formulate a generic approach to optimize NNS processes in accordance with production requirements and processes dynamics. In the previous investigations, NNS processes have been investigated on case-bycase basis, for example as in Gupta (2019).

Product design variation is limited by design constraints and working conditions, while process parameters are limited by technological feasibility and consequently strictly process dependant. Understanding their correlations across the process chain is fundamental for a holistic optimization of both process and product.

\section{Roadmap}

The next section reviews the literature on both numerical and design optimization. After the literature survey, the problem formulation is defined (section "Concurrent optimization of near net shape processes") and the proposed methodology (illustrated by a validation case study) is presented. The case study (section "Case study") requires optimisation of a blank (produced by centrifugal casting) and finishing machining steps (done by turning). To optimize the turning process, the literature is also reviewed to identify viable optimization's algorithm settings. Section "Case study results" presents the case study results, which suggest an overall cost saving of $22 \%$. Finally, section "Discussion" discusses the limitations of the CoDeO methodology before section "Conclusions" draws some conclusions.

\section{Literature survey: process and product design optimization for manufacturing processes}

Process optimization is a trade-off between maximizing the time performances and the process by considering the process dynamics. For this reason, the optimization model needs to take into consideration the process mechanics. In the literature, researchers introduce process models (e.g. in turning process: turning power, cutting force, obtainable roughness equations) into optimization algorithm in different ways: using these multi-variable equations (that often take the form of non-linear functions) as an objective function itself (i.e. having a multi-criteria optimization and therefore a Pareto solution), as non-linear constraints or in a combination of the previous.

The modelling of NNS processes depends on the optimization algorithm selected. In a multi-variable and multicriteria optimization, that NNS methods require, heuristics can be generally defined as search techniques that are able to provide, among several alternatives, the most effective solution for achieving a certain goal. The search based algorithm provides a rule, or set of rules, for identifying an acceptable solution, or solutions, at a certain (or acceptable) computational cost. Heuristic-based search techniques find viable application cases whenever conventional techniques fail to achieve results or their computational cost is too high (e.g. a high dimensional search space with several local optima). They have been widely used for combinatorial process optimization, providing near-optimal solution, or solutions. In these cases, researchers and practitioners prefer alternative cost effective near-optimal (or approximate) solution(s) rather than exact ones, as it may be extremely difficult to find the precise, optimal point in higher dimension, and multimodal search space (Mukherjee and Ray 2006).

Furthermore, optimization problem complexity has increased as the resolution of process measurement method have also improved. The availability of better measurement technology has resulted in discrete and continuous search spaces with multi-modal, differentiable as well as non-differentiable, objective functions or responses. 
The next section briefly reviews the main computational approaches researchers have adopted for multi-variable optimization in the NNS manufacturing and, generally, in primary shaping and machining process optimization. Generally, the different approaches to process optimization can be classified as conventional and non-conventional. While conventional techniques provide a local optimal solution, non-conventional techniques, which are based on extrinsic models or objective functions, are only approximations that aim to provide near-optimal condition. Conventional techniques can be classified in two categories: experimental and iterative mathematical search techniques. The first includes statistical design of experiment (e.g. Taguchi method) and response surface design methodology (RSM). On the other hand, iterative mathematical search techniques include linear programming (LP), non-linear programming (NLP), and dynamic programming (DP) algorithms. Non-conventional techniques include the meta-heuristic search based algorithm. Three main types of metaheuristic search based algorithm are applied in process parameters optimization: genetic algorithm (GA), simulated annealing (SA), and tabu search (TS).

Evolutionary algorithms have been found particularity effective in multi-objective optimization (Zitzler et al. 2000; Fonseca and Fleming 1995; Srinivas and Deb 1994; Bäck and Schwefel 1993) and, in particular, in manufacturing sector (Pierreval and Tautou 1997; Fonseca and Fleming 1998).

In the literature, evolutionary algorithms have been applied to the optimization of several different production systems and manufacturing processes. For example, evolutionary algorithms have been applied to optimize the performances of production (e.g. Aydoğan et al. 2019) and assembly lines (e.g. Minghai et al. 2019; Saif et al. 2019). Optimization techniques have also been widely applied to scheduling manufacturing problems, for example for taking into account random events (Fleury et al. 1999).

In particular, several authors apply and modify GAs for optimising conventional and non-conventional machining process. For example, general multi-criteria approaches are developed and used for optimizing turning, wire cutting and laser cutting by Rao et al. (2018). Dedicated evolutionary algorithms are developed and tested on specific processes such as water-jet (Rao et al. 2019) or grinding (Liu et al. 2019).

Evolutionary algorithms have been used for concurrently optimizing the product design and the correspondent manufacturing cost. In particular, authors, such as Ramesh et al. (2009), Al-Ansary and Deiab (1997) and Huang et al. (2005), focus on the optimal allocation of dimensional tolerances for product cost minimization using GAs and other evolutionary methods.

Several authors use evolutionary techniques for "on-line" process optimization. For example, Del Prete et al. (2019) use genetic programming for maximizing turning productivity (maximising removal rate) and reducing defect rate. Liu et al. (2019) compare Taguchi approach to non-linear methods in the context of on-line process control of injection moulding, assessing the superiority in performances of the latter. Similarly, Zainal et al. (2016) compare swarm optimization with Taguchi techniques for optimizing milling process.

Evolutionary optimization techniques have also been applied to design optimization in the early conceptual design phase, as in Burnell et al. (1991), Liu et al. (2019) and Lee and Wang (1992).

\section{Metaheuristic algorithms for manufacturing process and design optimization: application to near net shape processes}

Regarding NNS process, few authors attempt to associate the NNS concept to process chain optimization using evolutionary algorithms. Generally, the different investigation approaches are related to the process to be investigated. For example, Caporalli et al. (1998) investigate hot forging process, using numerical and experimental methodologies. Investigating two intercepting manufacturing lines (one producing a forging tool, another producing a workpiece though machining, annealing and forging). Their optimization model minimized the flow stress of every hot forging operation, using FEM simulation. Denkena et al. (2011) develop analytical and derived experimental models, minimizing a global cost function. The developed expression of the product quality is dependent on the tolerances achievable in machining and forging process. This formula is used concurrently with the total cost and leading time formulas for a multi-criteria optimization using Genetic Algorithms. Davidson et al. (2008) optimize a flow forming process using experimental trials, maximizing process quality (achievable tolerances) and optimizing microstructure using a Taguchi array (Design of Experiments). The optimization target is related to the process dynamics in the experimental approach. Sardinas et al. (2006) use a micro GA for optimizing a turning process by two objective functions: turning time and tool life. The algorithm uses two different populations: one static and one dynamic. The first is processed with the classic GA operators, creating the second population.

In an effort to improve the resolution and accuracy of machining process researchers have reported the application of many different computational models and algorithms. The optimization of process parameters is the key for economically efficient machining operations (Khan et al. 1997) because metal cutting is still a significant step in most manufacturing processes.

However these established processes need to respond to the increasing demand for quality, and so consequently 
optimization methods in metal cutting are vital tool for continuous improvement of process output quality. But finding optimal machining conditions is a complex problem and consequently metaheuristic searches (particularly the evolutionary algorithms) have been developed as "problem specific" solver, guiding, or modifying heuristics to produce optimal solutions. For example, Mukherjee and Ray (2006) provide the general applications advantages and disadvantages of evolutionary algorithm usage. Khan et al. (1997)) benchmark evolutionary algorithms, in particular GA and SA and gradients method, using different test function. GA and SA show consistency in converging to the global minima and are not influenced by the dimensions of the input vectors (i.e. variables number), although they increase the convergence time in comparison with differential methods. Regarding the differences between the specific models, SA gives high precision and the code can be run longer to get higher precision. For GA, the precision is proportional and limited by the precision used for representing each variable (i.e. number of bits). GA and SA advantages over gradient methods include the higher total number of function and constrains evaluable. Although, the higher functions evaluations per run (i.e. giving a high convergence time) is a disadvantage that make them unsuitable for real-time optimization.

In conclusion, GAs are most frequently used for single and multi-objective optimization of large (i.e. high number of variables) and complex problems (Deb 2001), because they:

- Do not require continuous or convex design space (as non-gradient based)

- Are able to explore large search space and have a high chance of avoiding local optima (due to is probabilistic and not deterministic nature)

- Provide multiple near-optimal solution.
- Are able to solve multiple objectives and non-linear response function problems, in both discrete and continuous cases.

Therefore, the GA is generally preferred when near-optimal condition(s) rather than exact optimal conditions are cost effective and acceptable for use by the manufacturers (Mukherjee and Ray 2006).

\section{Concurrent optimization of near net shape processes}

Problem formulation A generic NNS manufacturing chain can be modelled as a primary shaping process followed by a number of machining steps (Fig. 1). The primary shaping component can be either a forming, casting or additive layer manufacturing process. The industrial need is to have a simultaneous optimization of design and process parameters, achieving:

- Product geometric requirements (e.g. assembly constraints)

- Quantitative product requirements (e.g. surface roughness, component weight)

- Process quality (e.g. defect rate, tool wear, forging force)

- Production targets (e.g. product cost, production rate, lead-time)

The process parameters to consider are:

- Primary shaping process variables $\left(v_{1}, v_{2}, \ldots, v_{n}\right)$

- Machining process variables $\left(m_{11}, m_{12}, \ldots, m_{1 k}\right.$ $\left.m_{21}, m_{22}, \ldots, m_{2 k} m_{N 1}, m_{N 2}, \ldots, m_{N k}\right)$

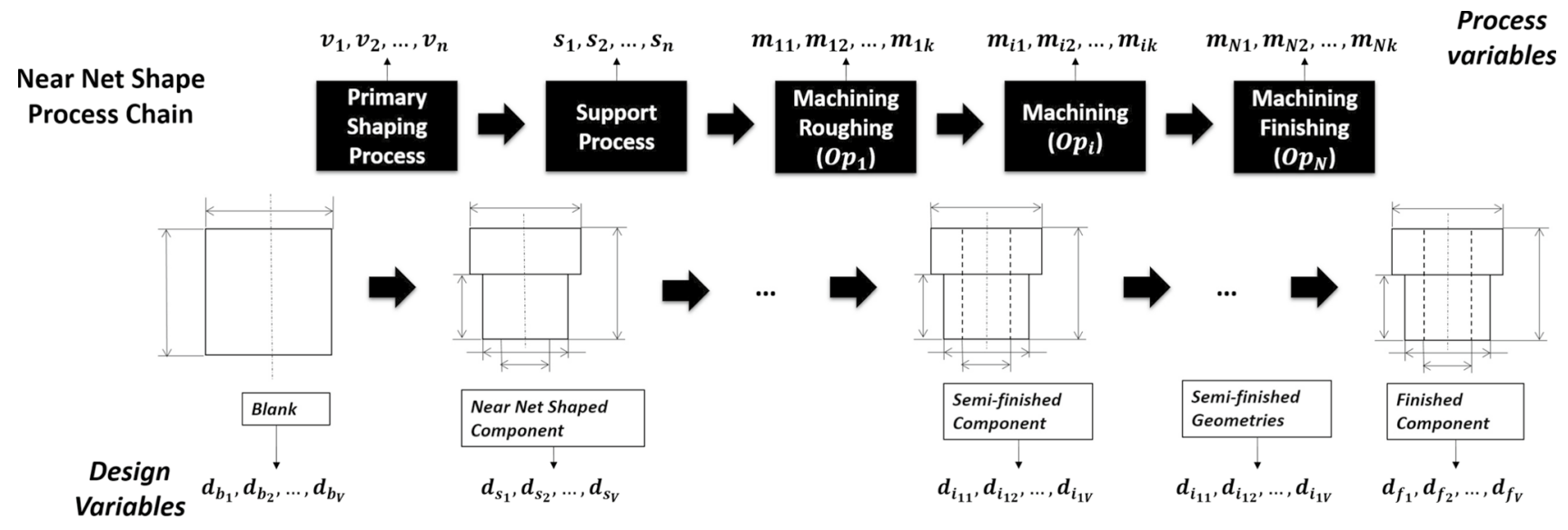

Fig. 1 Near net shape process optimization: schematic of variables and problem formulation 
where $N$ is the number of machining operations required, $n$ is the number of near net shape process variables, $k$ is the number of machining variables. Referring to Fig. 1, the design variables to consider are

- Billet, Preform or Raw Material Volume $\left(d_{b_{1}}, d_{b_{2}} \ldots d_{b_{V}}\right)$

- Near net shaped Component $\left(d_{s_{1}}, d_{s_{2}} \ldots d_{s_{V}}\right)$

- Intermediate machined geometries or Semi-finished component $\left(d_{i_{11}}, d_{i_{12}} \ldots d_{i_{1 V}} ; d_{i_{21}}, d_{i_{22}} \ldots d_{i_{2 V}} ; d_{i_{N 1}}, d_{i_{N 2}} \ldots d_{i_{N V}}\right)$

- Finished $\left(d_{f_{1}}, d_{f_{2}} \ldots d_{f_{V}}\right)$

where $V$ is the number of design variables.

Conditional design optimization ( $\mathrm{CoDeO}$ ) Figure 2 shows a schematic representation of the $\mathrm{CoDeO}$ methodology. The optimization method is associated with the primary shaping and machining process models. In general, for optimizing the product design and process parameters, it is necessary to have a cost model which is a function of both. The models needed for this optimization are:

1. Cost model of the primary shaping process, dependent on process parameters (e.g. forging force or intermediate geometry) and/or product design variables (e.g. diameter)

2. Cost model of the machining process dependent on process parameters and/or product design variables

3. Support models (i.e. linear or non-linear) for the primary shaping or machining processes (e.g. production rate target or turning force constraint)

The presence of support models is dependent on the nature of the processes, production targets and product requirements. A model can investigate the product quality (i.e. if it can be quantified by a function) or represent the technological aspect (e.g. forging force) to optimize (or keep within acceptable limits). By adding these as objectives function, the optimization changes from a single to a multi-criteria process. If these criteria can be added into other forms (e.g. non-linear constraints), the optimization can retain its single scope and optimising the cost by giving boundaries to the variable optimization.

These models allow the exploration of iterative systematic product and process design variation that can be optimized concurrently by minimizing the process cost (as main

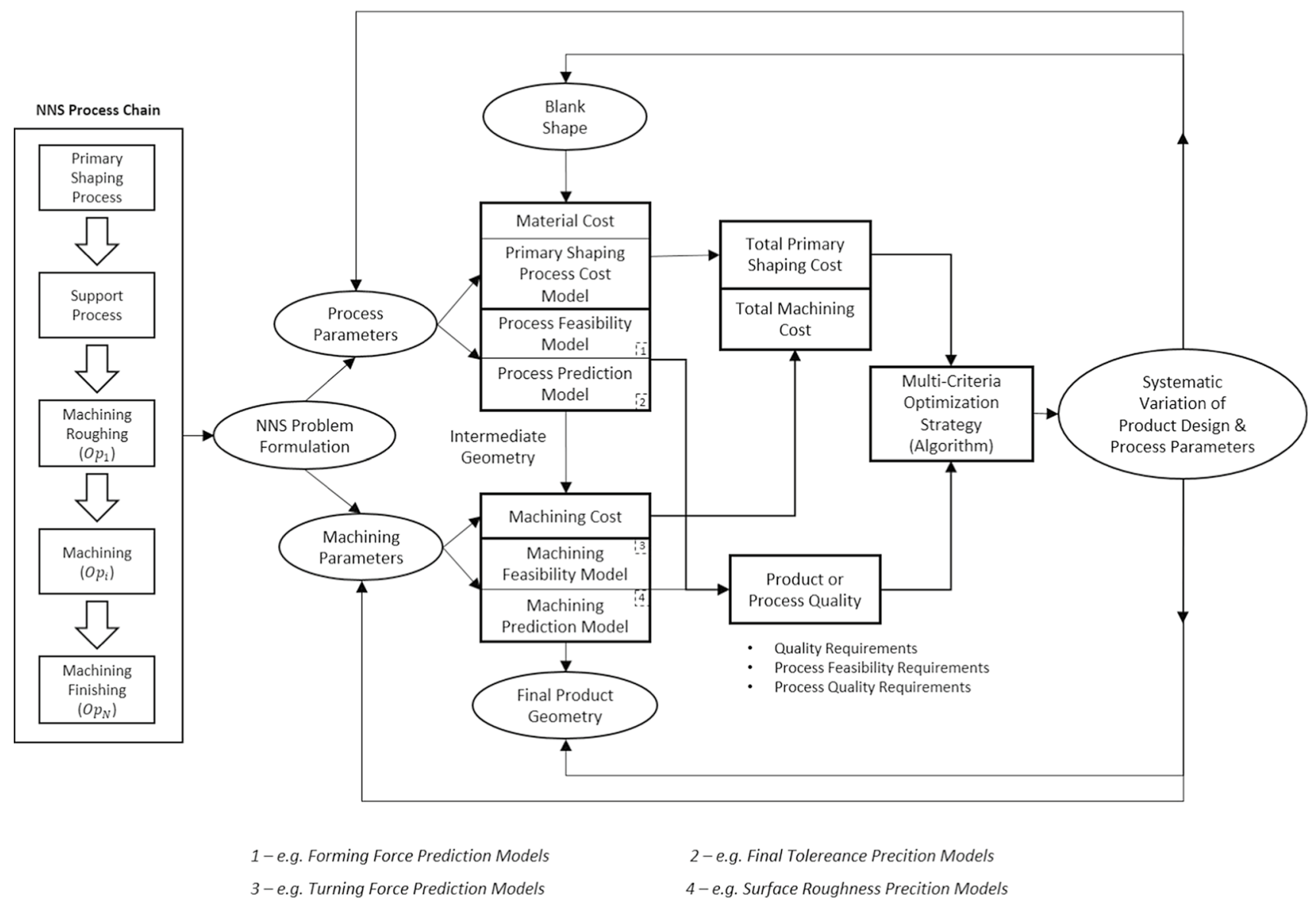

Fig. 2 Schematic of conditional design optimization $(\mathrm{CoDeO})$ methodology 
target),process technological features and product/production targets.

Referring to the Fig. 1 schematic, a machining process (milling or tuning) parameters can generally be synthesized in Eq. (1)

$m_{11}, m_{12}, \ldots, m_{1 k} ; m_{21}, m_{22}, \ldots, m_{2 k} ; m_{N 1}, m_{N 2}, \ldots, m_{N k}=a, v_{c}, F, N$

where, $a$ is the cutting depth, $v_{c}$ is the cutting speed $F$ is the feed rate, $N$ is number of passes.

Using geometrical constrains and machining parameters some of the intermediate machining geometries can be written as a function of the final and primary shaped geometries, by the number of machining steps (Eq. (2)).

$d_{i_{11}}, d_{i_{12}} \ldots d_{i_{1 N}}$

$d_{i_{21}}, d_{i_{22}} \ldots d_{i_{2 N}}$

$d_{i_{V 1}}, d_{i_{V 2}} \ldots d_{i_{V N}}=f\left(d_{s_{1}}, d_{s_{2}} \ldots d_{s_{V}}\right.$

$\left.d_{f_{1}}, d_{f_{2}} \ldots d_{f_{V}} ; a\right)=N$

This allows the number of machining step $N$ to be expressed as a function of the design variables.

Expressing the machining steps number as in Eq. (2), the total machining time $T_{M}$, and, consequently, the total machining $\operatorname{cost} C_{M}$, can be written as Eq. (3).

$T_{M}=f\left(d_{s_{1}}, d_{s_{2}} \ldots d_{s_{V}} ; d_{f_{1}}, d_{f_{2}} \ldots d_{f_{V}} ; a, v_{c}, F\right)$

In case of single criteria optimization, the objective function $O B J_{i}$ can be written as in Eq. (4)

$\left\{O B J_{1}=C_{N N S}+C_{M}=C N N S+T_{M} c_{M}\right.$

where, $c_{M}$ is the hourly machining cost and $C_{N N S}$ the cost model for the NNS process. In case of multi-criteria optimization, the objective functions can be formulated as in Eq. (5)

$\left\{\begin{array}{l}O B J_{1}=C_{N N S}+C_{M} \\ O B J_{2}=f\left(v_{1}, v_{2}, \ldots, v_{n}\right) \\ O B J_{3}=f\left(a, v_{c}, F\right)\end{array}\right.$

The cost model for the NNS process $C_{N N S}$ needs to be built individually, depending on the process nature and data available. Generally the cost is function of input and output geometries and process parameters.

$C_{N N S}=f\left(v_{1}, v_{2}, \ldots, v_{n} ; d_{b_{1}}, d_{b_{2}} \ldots d_{b_{V}} ; d_{s_{1}}, d_{s_{2}} \ldots d_{s_{V}}\right)$

Marini et al. (2017a) present a framework for technological and economic models building for near net shape process.

Non-linear constrains can be added to the objective functions, limiting the feasible solutions to those which match process limit capabilities (e.g. turning power constraint), quality targets (e.g. surface roughness constrain or product weight) and production targets (e.g. machining removal rate).

NNS process variables and models are dependent on the process nature, which could be established from literature or experimental investigation.

The design variables selection is similarly case dependant and can be influenced by several factors. The main constraints are usually geometrical or connected to key features for influencing component functionality or manufacturing costs however their definition is difficult to standardize.

The machining process variables are usually limited to the feed rate, cutting speed and cutting depth.

\section{Case study}

CoDeO has been applied to the optimization of the manufacturing of a control valve's cage, a complex component manufactured from 420 Stainless Steel. The schematic of a manufacturing chain for producing valve's component is showed in Fig. 3.

A centrifugal casting process and turning process are used for making the component. For brevity of presentation, the finishing turning and drilling processes are excluded from the optimization, although the finishing allowance is used for modeling the machining process (described in "Appendix B").

Variables definition: search space setting The optimization considers processes cost so consequently these need to be modelled into equations, optimizing both process parameters and design variables Fig. 4 displays the variables to be optimized and their collocation in the manufacturing chain and final/semi-finished products designs. Nine variables (defined in Table 1) have been considered for the optimization problem, as in Fig. 4: four process parameters and five design variables.

Using the centrifugal casting process parameters selection and modelling described in Marini and Corney (2017) and included in the cost equation (9), the diameter of the centrifugal castings internal cavity $\left(I D_{m}\right)$ is set in accordance to mould available and minimum required molten material for spinning. The final component dimensional variables $\left(O D, I D, O D_{b}, l_{f}, L_{f}\right)$ are informed by current assembly constraints and design targets. The cutting speed $\left(v_{c}\right)$, depth of cut $(a)$ and feed rate $(F)$ ranges are defined using (Kalpakjian and Schimd 2009) for medium and high C steels and ceramic coated carbide tool. This definitions include the values currently employed in the manufacturing process. The machine tool spindle speed $(n)$ is also used as a variable: its range derived from the cutting speed ranges and the minimum and maximum diameters. 

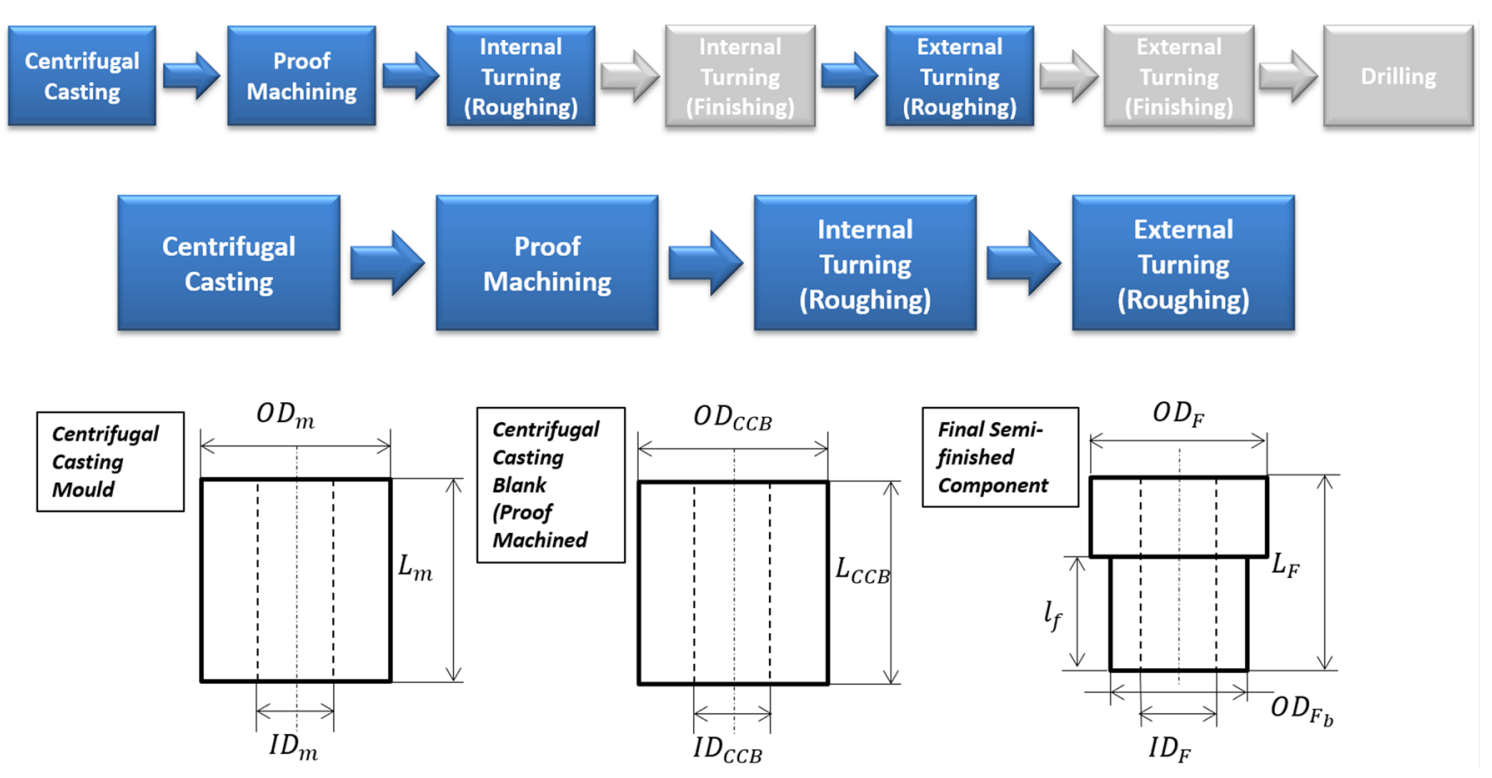

Fig. 3 Valve cage manufacturing process chain model for optimization

Table 1 Variable ranges

Fig. 4 Valve cage manufacturing optimization: schematic of process parameters and design variables

\begin{tabular}{lll}
\hline Variable & Min & Max \\
\hline$O D(\mathrm{~mm})$ & 480 & 500 \\
$I D(\mathrm{~mm})$ & 430 & 410 \\
$O D_{b}(\mathrm{~mm})$ & 460 & 480 \\
$L_{f}(\mathrm{~mm})$ & 415 & 425 \\
$l_{f}(\mathrm{~mm})$ & 50 & 70 \\
$a(\mathrm{~mm})$ & 2.5 & 7.6 \\
$n(\mathrm{RPM})$ & 80 & 261 \\
$F(\mathrm{~mm})$ & 0.15 & 0.75 \\
$I D_{m}(\mathrm{~mm})$ & 340 & 370 \\
\hline
\end{tabular}

To determine appropriate GA functions to support the optimization the literature was reviewed.

Literature survey: GA functions and settings for turing process optimization Many different applications for turning process optimization through metaheuristic search based algorithms have been reported in literature, in particular for GA. Cus and Balic (2003) use a GA search base algorithm for optimizing the turning process of steel. Saravanan et al. (2003) use a lower number of variables (i.e. feed rate and cutting speed) for testing turning process optimization through GA and SA. Amiolemhen and Ibhadode (2004) differentiate between roughing and finishing operations, introducing different unit production cost fitness function and use different constrains for every analysed operation. Sardinas et al. (2006) use a micro GA for optimizing a turning process by two objective functions: turning time and tool life. The
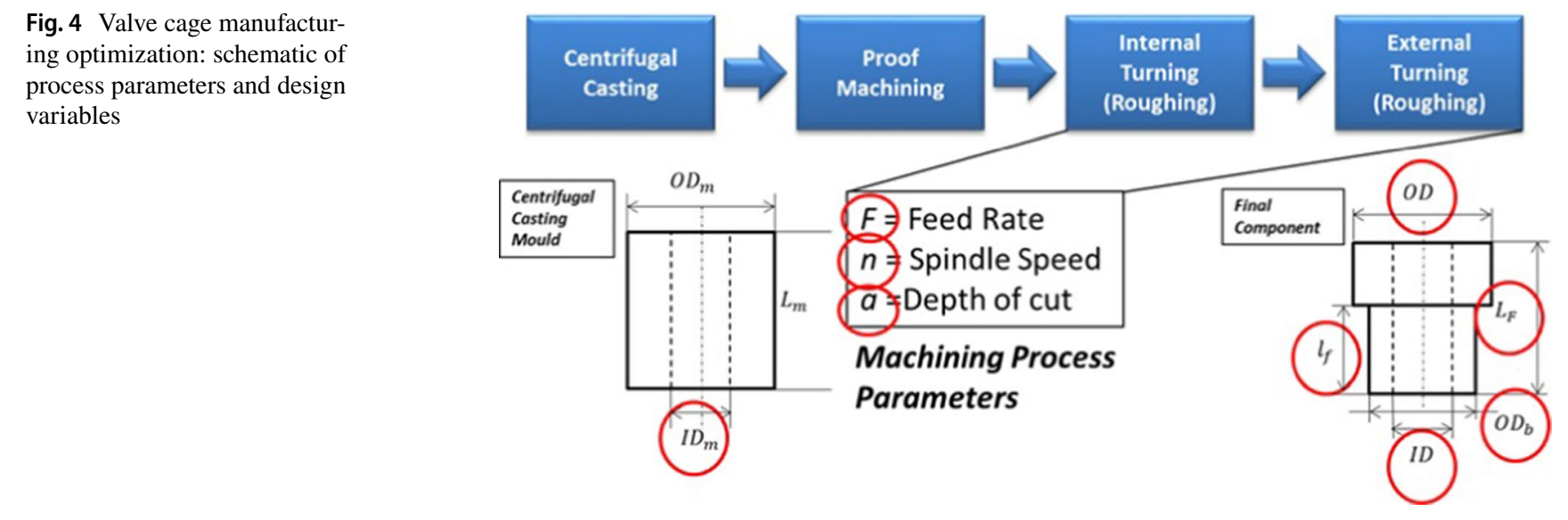
first is processes with the classic GA operators, creating the second. This new individuals are added to the static ones in the next iteration, meanwhile the other population remain unchanged, even if they violate the constraints (measured by the violation grade by an unfeasibility index). Yildiz and Ozturk (2006) propose an integration of Taguchi robust design optimization (i.e. using S/N ratio and ANOVA) and GA. The authors test the computational power of single and multi-criteria optimization through this hybrid algorithm, comparing it with previous hybrid GA in literature. Yang and Natarajan (2010) compare a multi-objective differential evolution algorithm and non-dominated sorting genetic algorithm for the turning optimization of a particular tool (steel and tungsten carbide). The differential evolution (DE) algorithm acts on GA by narrowing down its search space during each step. D'Addona and Teti (2013) use the MATLAB GA optimization toolbox for optimizing the turning process of cast steel blank with an HSS tool, comparing two crossover operators (single point and two-points) and mutation coefficients.

Tables 2 and 3 summarize the approaches proposed for optimization of single pass turning process. Most of the authors use only the main turning process parameters as variables (feed rate, cutting speed and depth of cut). Similarly, all the authors used the unit production cost as fitness function (or as a part of it). Similar settings are applied also in the crossover and mutation fractions. All the authors include linear and non-linear constraints for limiting the individuals' feasibility.

For multi-passes turning, the researchers tend to use iterative cycles, optimizing each pass individually, usually, having every pass interacting with the previous and next ones. So essentially, the authors are applying single objective optimization, using the unit process cost (Onwubolu and Kumalo 2001; Yildiz 2013; Alberti and Perrone 1999; Wang et al. 2002) or customized linear function combining tool life and cost (Schrader 2003).

Objective functions Having determined that the multicriteria optimization method will be a GA (section "Concurrent optimization of near net shape processes"), two objective functions must be defined. The first objective function (Eq. (7)) is the total cost (i.e. including centrifugal casting and machining cost). The second fitness function is the turning force (Eq. (8)), developed by Sardinas et al. (2006). The two functions are:

1. Total cost model

$$
F F_{1}=C_{C C B}+\left(T_{t u}\right) c_{m}
$$

Table 2 Summary of GA literature on turning process (Part I)

\begin{tabular}{|c|c|c|c|}
\hline GA characteristics & Cus and Balic (2003) & Saravanan et al. (2003) & Amiolemhen and Ibhadode (2004) \\
\hline Fitness function(s) number & One & One & One \\
\hline Minimization objective(s) & $\begin{array}{l}\text { Exponential function (combining } \\
\text { machining removal rate, operation } \\
\text { cost, surface roughness) }\end{array}$ & Unit production cost & Unit production cost \\
\hline Minimization function(s) Type & Analytical & Analytical & Analytical \\
\hline Variables number & 3 & 2 & 3 \\
\hline Variables & $\begin{array}{l}\text { Depth of cut }(a) \text {, feed rate }(F), \text { cut- } \\
\text { ting speed }(v)\end{array}$ & Feed rate $(\mathrm{F})$, cutting speed $(\mathrm{v})$ & $\begin{array}{l}\text { Depth of cut }(\mathrm{d}) \text {, feed rate }(\mathrm{F}) \text {, cut- } \\
\text { ting speed }(\mathrm{v})\end{array}$ \\
\hline $\begin{array}{l}\text { Constrain functions (linear-nonLin- } \\
\text { ear functions) }\end{array}$ & $\begin{array}{l}\text { Cutting force (An), cutting power } \\
\text { (An) }\end{array}$ & $\begin{array}{l}\text { Cutting force (An), cutting } \\
\text { power (An), tool tip tem- } \\
\text { perature }\end{array}$ & $\begin{array}{l}\text { Tool life (An), cutting force (An), } \\
\text { cutting power (An), Chip-tool } \\
\text { interface temperature (An), } \\
\text { dimensional accuracy (An), cut- } \\
\text { ting region stability (An), surface } \\
\text { finish (An) }\end{array}$ \\
\hline Initial population: coding type & Not known & Binary coding (bit string) & Binary coding (bit string) \\
\hline $\begin{array}{l}\text { Initial population: size (number of } \\
\text { individuals) }\end{array}$ & Not known & 20 & 20 \\
\hline Initial population creation function & Not known & Random generation & Random generation \\
\hline Reproduction: selection function & Not known & Rank order & Roulette wheel \\
\hline Reproduction: elite count (fraction) & Not known & None & None \\
\hline Crossover: function & Not known & One-point operator & One-point operator \\
\hline Crossover: fraction & Not known & 0.8 & 0.8 \\
\hline Mutation: fraction & Not known & 0.01 & 0.01 \\
\hline $\begin{array}{l}\text { Supplementary applied optimiza- } \\
\text { tion techniques }\end{array}$ & None & Simulated annealing & None \\
\hline
\end{tabular}


Table 3 Summary of GA literature on turning process (Part II)

\begin{tabular}{|c|c|c|c|c|}
\hline GA characteristics & Yildiz and Ozturk (2006) & Sardinas et al. (2006) & Yang and Natarajan (2010) & D’Addona and Teti (2013) \\
\hline Fitness function(s) number & One (two) & Two & One & One \\
\hline Minimization objective(s) & Unit Production Cost & $\begin{array}{l}\text { Unit production time, tool } \\
\text { life }\end{array}$ & Linear function & Unit production time \\
\hline $\begin{array}{l}\text { Minimization function(s) } \\
\text { type }\end{array}$ & Analytical & Cost (An), tool life (Ex) & $\begin{array}{l}\text { Machining removal rate, } \\
\text { (An), Tool wear (Ex) }\end{array}$ & Analytical \\
\hline Variables number & 5 & 3 & 3 & 3 \\
\hline Variables & $\begin{array}{l}\text { Depth of cut, feed rate, } \\
\text { cutting speed, number of } \\
\text { rough cuts }\end{array}$ & $\begin{array}{l}\text { Depth of cut (a), feed rate } \\
(\mathrm{F}) \text {, cutting speed (v) }\end{array}$ & $\begin{array}{l}\text { Depth of cut (a), feed rate } \\
(\mathrm{F}) \text {, cutting speed (v) }\end{array}$ & $\begin{array}{l}\text { Depth of cut (a), feed rate } \\
(\mathrm{F}) \text {, cutting speed (v) }\end{array}$ \\
\hline $\begin{array}{l}\text { Constrain functions (linear- } \\
\text { nonLinear functions) }\end{array}$ & $\begin{array}{l}\text { Cutting force (An), tool- } \\
\text { life (An), cutting region } \\
\text { stability (An), Surface } \\
\text { finish (An) [for both } \\
\text { finishing and roughing] }\end{array}$ & $\begin{array}{l}\text { Cutting force }(\mathrm{Ex}) \text {, cutting } \\
\text { power }(\mathrm{Ex}), \text { tool life } \\
(\mathrm{Ex}), \text { surface finish }(\mathrm{An})\end{array}$ & $\begin{array}{l}\text { Surface roughness } \\
(\mathrm{Ex}) \text {, cutting zone } \\
\text { temperature }(\mathrm{Ex})\end{array}$ & $\begin{array}{l}\text { Cutting force (An), cutting } \\
\text { power (An), tool life } \\
(A n), \text { surface finish }(A n)\end{array}$ \\
\hline $\begin{array}{l}\text { Initial population: coding } \\
\text { type }\end{array}$ & Binary coding (bit string) & Binary coding (bit string) & Binary coding (bit string) & Binary coding (bit string) \\
\hline $\begin{array}{l}\text { Initial population: size } \\
\text { (number of individuals) }\end{array}$ & 80 & 500 static (10 dynamic) & 100 & $20-100$ \\
\hline $\begin{array}{l}\text { Initial population creation } \\
\text { function }\end{array}$ & Random generation & Random generation & Random generation & Random generation \\
\hline $\begin{array}{l}\text { Reproduction: selection } \\
\text { function }\end{array}$ & Unknown & Tournament Selection & Tournament selection & $\begin{array}{l}\text { MATLAB default (stochas- } \\
\text { tic uniform) }\end{array}$ \\
\hline $\begin{array}{l}\text { Reproduction: elite count } \\
\text { (fraction) }\end{array}$ & None & 0.25 & Adaptable (DE) & $\begin{array}{l}\text { MATLAB default } \\
(0.2 * \text { Population size })\end{array}$ \\
\hline Crossover: function & Unknown & Two-points operator & Ranking (DE) & $\begin{array}{l}\text { Single point (two-points) } \\
\text { operator }\end{array}$ \\
\hline Crossover: fraction & 0.9 & Not Known & 0.9 & 0.8 \\
\hline Mutation: fraction & 0.5 & 0.0001 & 0.1 & 0.1 \\
\hline $\begin{array}{l}\text { Supplementary applied } \\
\text { optimization techniques }\end{array}$ & $\begin{array}{l}\text { Taguchi robust param- } \\
\text { eter design (S/N ratio, } \\
\text { ANOVA) }\end{array}$ & $\begin{array}{l}\text { Dynamic GA approach } \\
\text { (micro-GA) }\end{array}$ & $\begin{array}{l}\text { Differential evolution (DE) } \\
\text { algorithm }\end{array}$ & None \\
\hline
\end{tabular}

2. Turning cutting force, as in Sardinas et al. (2006)

$$
\begin{aligned}
F F_{2} & =F_{T}=\frac{6.56\left(10^{3}\right) F^{0.917} a^{1.1}}{v_{c}^{0.286}} \\
& =\frac{6.56\left(10^{3}\right) F^{0.917} a^{1.1}}{\left(\frac{O D n 3.14}{1000}\right)^{0.286}}
\end{aligned}
$$

Centrifugal casting model The first part of Eq. (7) refers to the centrifugal casting blank cost $\left(C_{C C B}\right)$, which is defined by the Eq. (9)

$$
\begin{aligned}
& C_{C C B}=\left(\left(O D_{m}^{2}-I D_{m}^{2}\right) K_{c}\right. \\
& \left.-\left(O D_{C C B}^{2}-I D_{C C B}^{2}\right) K_{v}\right) \rho_{m} \frac{L_{f}}{4} \pi 10^{6}
\end{aligned}
$$

With, $O D_{m}$ the mould outer diameter and $L_{f}$ mould length. The selection of $O D_{m}$ and $I D_{m}$ from the target casting dimension, and the analytical relationship with $I D_{m}$ are detailed in Marini and Corney (2017). $K_{c}$ and $K_{v}$ constants value, depending on the centrifugal casting operation, considered 5.56 and 3.17 respectively for the selected material and mould external diameter (Marini and Corney 2017).

Turning model The second part of Eq. (7) refers turning cost, which is proportional to the turning time $\left(t_{i_{T}}\right)$ and the cost per hour $\left(c_{m}\right)$. The total turning cost is dependent on the machining strategy, showed in Fig. 5). The turning process is divided into three main operations, which correspond to three different machining time per pass $\left(T_{T u}\right)$. The total turning time can be written as:

$T_{T u}=t_{I N_{T}}+t_{E X_{T}}+t_{E X_{b T}}$

With, $t_{E X_{T}}$, external turning time; $t_{E X_{b T}}$ : external bottom turning time; $t_{I N_{T}}$, internal turning time (Fig. 5).

The turning process has been analysed as a helicoidal motion, resembling the cutting trajectory, instead of a linear one (i.e. classic turning formulation), resembling the tool motion. The aim of this new formulation is to gather the process parameters, diameters and number of passes in a 
Fig. 5 Turning strategy schematic: external turning zone (red); external bottom section turning (yellow), internal turning (green) (Color figure online)

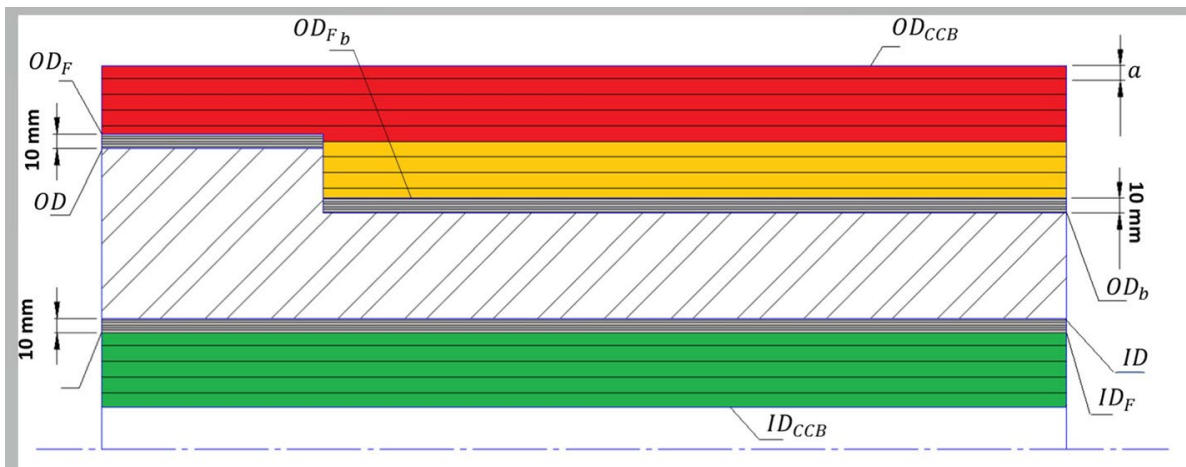

single formulation, for a single iteration optimization. The new formulation results equivalent to classic formulation. The derivation of the turning time formula is showed in "Appendix A".The generic helix turning time can be written as in Eq. 11.

$t_{T}=\sum_{i=1}^{N} t_{i}=\frac{60 N L}{n} \sqrt{\frac{1+\pi^{2} \sum_{i=1}^{N} D_{i}}{F^{2}+\pi^{2} \sum_{i=1}^{N} D_{i}}}$

In this way, the model takes into consideration the turning process dynamics when optimizing the process parameters and the design variables. The solution is consequently a trade-off between process time minimization (thus turning cost) and the cutting force minimization.

Non-linear constraints According to the literature, nonlinear constrains have been add to the GA, limiting the feasible individuals to matching the turning process limit capabilities (i.e. turning power), quality targets (i.e. surface roughness) and production targets (machining removal rate). Turning power (Eq. (12), used in Sardinas et al. (2006); Cus and Balic (2003); Saravanan et al. (2003); Amiolemhen and Ibhadode (2004); Yildiz (2013)) and surface roughness (Eq. (13), used in Sardinas et al. (2006); Amiolemhen and Ibhadode (2004); Yildiz (2013); D'Addona and Teti (2013); Yang and Natarajan (2010))are used as non-linear inequality constraints for the machining parameters. The machining removal rate constrain (Eq. (14)) ensure the required production volume by a machining parameters feasible setting (i.e. previous constraints and the "traded-off" by the turning force fitness function). The non-linear constraints are showed as follows.

1. Turning power $\left(W_{T u}\right)$

$$
W_{T u}=\left(n O D \frac{3.14}{1000}\right) a F \frac{k_{c}}{60 * 10^{3}}<W_{M a x}
$$

With, maximum turning Power $\left(W_{\text {Max }}\right)$ of $82 \mathrm{~kW}$, correspondent to the target machine capabilities

2. Surface roughness $\left(R a_{T u}\right)$

$$
R a_{T u}=\left(\frac{F^{2}}{8 r_{T N}}\right) 1000<R a_{M a x}
$$

With, maximum Roughness $\left(R a_{M a x}\right)$ of $12.4 \mu m$, correspondent to the target component roughness.

3. Machining Removal Rate (MRR)

$$
M R R=\pi O D n F a>M R R_{\text {Min }}
$$

With, minimum machining removal rate higher $\left(M R R_{M i n}\right)$ higher than the current one $\left(1.3910^{5} \mathrm{~mm}^{3} / \mathrm{min}\right.$ ) .

The GA settings are summarized in Table 4

\section{Case study results}

The two objective functions (Equations (7) and (8)) are concurrent and the solution form a Pareto frontier. Accordingly to the machining parameters increase, the cutting force increase, remaining into feasible ranges. The surface roughness improvements cause the turning power to increase. The largest improvements are seen in the machining removal rate and, so, the machining time and cost decreases as a consequence.

Regarding the optimized variables, it is possible to notice a general reduction of the final dimensions, with the exception of the bottom section length, which increases. The machining parameters can be set higher than those currently applied ones (except from the feed rate that remaining unchanged). The following observations can be made

1. Design parameters vary in the first generations before converging over an optimal set of variables (constant for all the last generation individuals).

2. Casting process parameter behave similarly to the design parameters (connected by geometrical relationships).

3. Feed rate reach an optimal constant value.

4. Depth of cut and spindle speed vary in every solution. 
Table 4 GA settings

\begin{tabular}{ll}
\hline Fitness functions number & Two (multi-objective optimization) \\
Fitness parameter(s) & Unit production cost (FFI), cutting force (FFII) \\
Coding type & Binary coding (bit string) \\
Variables number & 9 \\
Variables & Centrifugal casting parameters: Casting mould internal diameter \\
& Turning parameters: feed rate, cutting speed, depth of cut \\
& Product design variables: outer diameter, outer bottom section \\
& diameter, internal diameter, bottom section length, total length \\
Non-linear constraints & Turning power, surface roughness, machining removal rate \\
Population & Size (number of individuals): 100 \\
& Creation function: random generation \\
& Selection function: tournament selection \\
Reproduction & Elite count: $0.05 \%$ population size \\
Crossover & Function: one-point operator \\
& Function: scattered crossover \\
Mutation & Fraction: 0.8 \\
Termination criteria & Function: adaptive feasible mutation \\
\hline
\end{tabular}

5. Total cost varies and remain coherent with current total cost

6. Cutting force and turning power vary congruently with machining parameters

7. Surface roughness varies with the machining parameters, satisfying the target requirements.

8. Machining removal rate varies with the machining parameters, although it remains sensibly higher than the current rate (average of $5.2810^{5} \mathrm{~mm}^{3} / \mathrm{min}$ ).

9. Similarly, machining time varies with machining parameters, remaining lower than current value $(75 \mathrm{~min}$ on average).

Tables 5 and 6 show the GA results, displaying optimized set of variables (fittest individuals) and resulting constraints, objectives functions and performances. Considering the average results of the optimal variable sets, the optimized geometry and process parameters adoption show an improvement in performances. Optimized machining parameters gives up to a 9.5 times higher removal rate. Using centrifugal cast blank, the machining time decreases between $8.3 \%$ and $21.6 \%$. The latter process configuration has been applied by the manufacturers and resulted in a cost saving of 22\% (The Weir Group PLC 2015).

Considering the selected configuration (Table 7), the improvement of process performances (i.e. interaction between process parameters and design variables) combined with the adoption of optimal design variables resulted in a final weight reduction of $10 \%$. This $19 \mathrm{~kg}$ material wastage reduction resulted in an estimated 2.8 ton scrap reduction per year (The Weir Group PLC 2015).

\section{Discussion}

Many reported NNS analytical approaches aim to optimize cost (Denkena et al. 2011; Sardinas et al. 2006), while the empirical experimental approaches tend to maximize process quality (Caporalli et al. 1998; Davidson et al. 2008). CoDeO optimize concurrently the manufacturing chain's cost and process feasibility, depending on analysed process characteristics and global cost model. For example, turning force has been used as trade-off (i.e. Pareto optimization) for the multi-criteria optimization of turning process using Genetic Algorithm. Similarly to Sardinas et al. (2006), The minimization of turning force makes the turning operation more feasible, as the minimization of costs tended to increase the machining parameters.

$\mathrm{CoDeO}$ is not able to use tool and equipment as design variable (unlike Caporalli et al. 1998's expert system that optimize the forming die shape and characteristics). On the other hand, CoDeO can optimize simultaneously process parameters and design variables.

In contrast with other NNS approaches (Caporalli et al. 1998; Denkena et al. 2011), CoDeO optimization can be adapted to different NNS processes.

In comparison with other concurrent optimization approaches, CoDeO achieves similar optimization targets to the methodologies used for robotic systems ( $\mathrm{Bi}$ and Zhang 2001; Li et al. 2001; Rampersad 1995; Ouyang et al. 2004) in terms of process parameters and design (both subject to design constraints). CoDeO is not an "offline" methodology and its scope is limited to the concurrent 
Table 5 GA results: part I

\begin{tabular}{|c|c|c|c|c|c|c|c|c|c|}
\hline Results & $\mathrm{OD}[\mathrm{mm}]$ & $\mathrm{ID}[\mathrm{mm}]$ & $\mathrm{a}[\mathrm{mm}]$ & $\mathrm{n}[\mathrm{RPM}]$ & $\mathrm{F}[\mathrm{mm} / 1]$ & $O D_{b}$ & $l_{f}$ & $L_{f}$ & $I D_{m}$ \\
\hline Individual 1 & 495.5 & 410.5 & 7.3 & 254.0 & 0.25 & 477.7 & 65.7 & 415.0 & 370.0 \\
\hline Individual 2 & 495.5 & 410.5 & 7.3 & 254.0 & 0.25 & 477.8 & 65.7 & 415.0 & 370.0 \\
\hline Individual 3 & 499.2 & 410.6 & 6.2 & 254.0 & 0.23 & 477.8 & 65.7 & 415.0 & 370.0 \\
\hline Individual 4 & 499.3 & 410.5 & 6.2 & 254.0 & 0.18 & 477.8 & 65.7 & 415.0 & 370.0 \\
\hline Individual 5 & 499.2 & 410.6 & 6.2 & 254.0 & 0.23 & 477.8 & 65.7 & 415.0 & 370.0 \\
\hline Individual 6 & 499.2 & 410.5 & 6.2 & 253.9 & 0.15 & 477.7 & 65.7 & 415.0 & 370.0 \\
\hline Individual 7 & 499.4 & 410.5 & 5.6 & 254.0 & 0.16 & 477.8 & 65.7 & 415.0 & 370.0 \\
\hline Individual 8 & 495.5 & 410.9 & 7.1 & 254.0 & 0.24 & 477.7 & 65.5 & 415.0 & 370.0 \\
\hline Individual 9 & 499.2 & 410.7 & 6.2 & 254.0 & 0.20 & 477.7 & 65.7 & 415.0 & 370.0 \\
\hline Individual 10 & 495.5 & 410.5 & 7.1 & 254.0 & 0.25 & 477.7 & 65.7 & 415.0 & 370.0 \\
\hline Individual 11 & 499.3 & 410.5 & 6.2 & 254.0 & 0.21 & 477.8 & 65.7 & 415.0 & 370.0 \\
\hline Individual 12 & 499.2 & 410.5 & 6.2 & 254.0 & 0.17 & 477.7 & 65.7 & 415.0 & 370.0 \\
\hline Individual 13 & 499.2 & 410.5 & 6.2 & 254.0 & 0.17 & 477.7 & 65.7 & 415.0 & 370.0 \\
\hline Individual 14 & 499.2 & 410.5 & 6.2 & 253.9 & 0.15 & 477.7 & 65.7 & 415.0 & 370.0 \\
\hline Individual 15 & 499.2 & 410.5 & 6.2 & 254.0 & 0.18 & 477.7 & 65.7 & 415.0 & 370.0 \\
\hline Individual 16 & 495.5 & 410.5 & 7.3 & 254.0 & 0.16 & 477.8 & 65.7 & 415.0 & 370.0 \\
\hline Individual 17 & 495.5 & 410.5 & 7.3 & 254.0 & 0.16 & 477.8 & 65.7 & 415.0 & 370.0 \\
\hline Individual 18 & 499.4 & 410.5 & 6.2 & 254.0 & 0.24 & 477.9 & 65.5 & 415.0 & 370.0 \\
\hline Individual 19 & 499.3 & 410.6 & 6.2 & 254.0 & 0.16 & 477.8 & 65.7 & 415.0 & 370.0 \\
\hline Individual 20 & 495.5 & 410.6 & 7.1 & 254.0 & 0.23 & 477.8 & 65.7 & 415.0 & 370.0 \\
\hline Individual 21 & 499.3 & 410.5 & 6.2 & 254.0 & 0.21 & 477.8 & 65.7 & 415.0 & 370.0 \\
\hline Individual 22 & 499.2 & 410.5 & 6.2 & 253.9 & 0.23 & 477.8 & 65.7 & 415.0 & 370.0 \\
\hline Individual 23 & 495.5 & 410.6 & 7.1 & 254.0 & 0.23 & 477.8 & 65.7 & 415.0 & 370.0 \\
\hline Individual 24 & 495.5 & 410.5 & 7.1 & 253.9 & 0.22 & 477.7 & 65.7 & 415.0 & 370.0 \\
\hline Individual 25 & 495.5 & 410.5 & 7.1 & 254.0 & 0.19 & 477.7 & 65.7 & 415.0 & 370.0 \\
\hline Individual 26 & 499.3 & 410.5 & 6.2 & 254.0 & 0.16 & 477.8 & 65.7 & 415.0 & 370.0 \\
\hline Individual 27 & 495.5 & 410.5 & 5.6 & 254.0 & 0.16 & 477.7 & 65.5 & 415.0 & 370.0 \\
\hline Individual 28 & 499.3 & 410.5 & 6.2 & 254.0 & 0.18 & 477.8 & 65.7 & 415.0 & 370.0 \\
\hline Individual 29 & 499.3 & 410.7 & 6.2 & 254.0 & 0.25 & 477.8 & 65.7 & 415.0 & 370.0 \\
\hline Individual 30 & 495.5 & 410.6 & 7.1 & 254.0 & 0.22 & 477.8 & 65.7 & 415.0 & 370.0 \\
\hline Individual 31 & 499.3 & 410.5 & 6.2 & 253.9 & 0.19 & 477.8 & 65.7 & 415.0 & 370.0 \\
\hline Individual 32 & 495.5 & 410.5 & 7.1 & 254.0 & 0.21 & 477.8 & 65.7 & 415.0 & 370.0 \\
\hline Individual 33 & 495.5 & 410.5 & 5.6 & 253.9 & 0.16 & 477.8 & 65.7 & 415.0 & 370.0 \\
\hline Individual 34 & 495.5 & 410.9 & 7.1 & 254.0 & 0.23 & 477.9 & 65.5 & 415.0 & 370.0 \\
\hline Individual 35 & 499.3 & 410.5 & 6.2 & 254.0 & 0.22 & 477.8 & 65.7 & 415.0 & 370.0 \\
\hline
\end{tabular}

optimization of the product design and process parameters of NNS manufacturing chains. In order words it is unable to influence the manufacturing control system ("on-line"). While the Design for Control methodologies are designed for the flexible manufacturing systems, CoDeO application is limited to NNS processes. However, CoDeO is able to consider a broader range of primary shaping processes (from sand casting to additive manufacturing) and different modelling methods (e.g. experimentally derived models).

Although $\mathrm{CoDeO}$ has flexibility in the manufacturing processes and product designs considered. Its search space is limited in comparison to optimization methods that integrate both design and operations management (Zhang et al. 2019; Ha and Porteus 1995; Zhang and Wang 2016). Other researchers have tried to generalize the modelling of entire manufacturing ecosystem (Zhang et al. 2019). Their frameworks take a global approach to the enterprise and supply chain, including the product design and operational phases.

While the resulting models are more flexible and holistic, their implementation is more complex as an additional phase of abstraction is required for utilizing their models in practice. In contrast, $\mathrm{CoDeO}$ limits its scope to a particular manufacturing sub-system of the whole production system (Giachetti 1998), although modelling it with higher accuracy.

$\mathrm{CoDeO}$ can be integrated with this general methodology in order to provide mathematical modelling and concurrent optimization of NNS manufacturing chains. 
Table 6 GA results: part II

\begin{tabular}{|c|c|c|c|c|c|c|c|c|}
\hline Results & Total cost $[£]$ & Cutting force $[\mathrm{N}]$ & Power [kW] & $\operatorname{MRR}\left[\mathrm{mm}^{3} / \mathrm{min}\right]$ & $\begin{array}{l}\text { Rough- } \\
\text { ness [Ra] }\end{array}$ & $\begin{array}{l}\text { Machining } \\
\text { time [min] }\end{array}$ & $\begin{array}{l}\text { Machining } \\
\text { cost }[£]\end{array}$ & $\begin{array}{l}\text { Casting } \\
\text { blank cost } \\
{[£]}\end{array}$ \\
\hline Individual 1 & 2124 & 2950 & 25.1 & $7.2 \mathrm{E}+05$ & 3.9 & 56 & 80 & 1302 \\
\hline Individual 2 & 2124 & 2930 & 25.0 & $7.1 \mathrm{E}+05$ & 3.9 & 56 & 80 & 1302 \\
\hline Individual 3 & 2140 & 2290 & 19.9 & $5.7 \mathrm{E}+05$ & 3.4 & 66 & 94 & 1302 \\
\hline Individual 4 & 2163 & 1772 & 15.1 & $4.3 \mathrm{E}+05$ & 1.9 & 87 & 125 & 1302 \\
\hline Individual 5 & 2142 & 2244 & 19.5 & $5.6 \mathrm{E}+05$ & 3.2 & 68 & 97 & 1302 \\
\hline Individual 6 & 2180 & 1538 & 12.9 & $3.7 \mathrm{E}+05$ & 1.4 & 102 & 146 & 1302 \\
\hline Individual 7 & 2205 & 1449 & 12.3 & $3.5 \mathrm{E}+05$ & 1.6 & 97 & 139 & 1302 \\
\hline Individual 8 & 2127 & 2797 & 23.8 & $6.8 \mathrm{E}+05$ & 3.7 & 58 & 82 & 1302 \\
\hline Individual 9 & 2152 & 2031 & 17.4 & $5.0 \mathrm{E}+05$ & 2.6 & 76 & 108 & 1302 \\
\hline Individual 10 & 2125 & 2879 & 24.6 & $7.0 \mathrm{E}+05$ & 3.9 & 56 & 80 & 1302 \\
\hline Individual 11 & 2147 & 2107 & 18.2 & $5.2 \mathrm{E}+05$ & 2.8 & 72 & 103 & 1302 \\
\hline Individual 12 & 2167 & 1713 & 14.5 & $4.1 \mathrm{E}+05$ & 1.8 & 91 & 130 & 1302 \\
\hline Individual 13 & 2170 & 1677 & 14.2 & $4.0 \mathrm{E}+05$ & 1.7 & 93 & 133 & 1302 \\
\hline Individual 14 & 2180 & 1538 & 12.9 & $3.7 \mathrm{E}+05$ & 1.4 & 102 & 146 & 1302 \\
\hline Individual 15 & 2160 & 1837 & 15.7 & $4.5 \mathrm{E}+05$ & 2.1 & 84 & 120 & 1302 \\
\hline Individual 16 & 2158 & 1932 & 15.8 & $4.5 \mathrm{E}+05$ & 1.6 & 88 & 126 & 1302 \\
\hline Individual 17 & 2155 & 1984 & 16.3 & 4.7E+05 & 1.6 & 86 & 123 & 1302 \\
\hline Individual 18 & 2137 & 2376 & 20.7 & $5.9 \mathrm{E}+05$ & 3.7 & 63 & 91 & 1302 \\
\hline Individual 19 & 2172 & 1649 & 13.9 & $4.0 \mathrm{E}+05$ & 1.6 & 94 & 135 & 1302 \\
\hline Individual 20 & 2131 & 2640 & 22.3 & $6.4 \mathrm{E}+05$ & 3.2 & 62 & 88 & 1302 \\
\hline Individual 21 & 2149 & 2060 & 17.7 & $5.1 \mathrm{E}+05$ & 2.6 & 74 & 106 & 1302 \\
\hline Individual 22 & 2139 & 2325 & 20.2 & $5.8 \mathrm{E}+05$ & 3.4 & 65 & 93 & 1302 \\
\hline Individual 23 & 2129 & 2712 & 23.0 & $6.6 \mathrm{E}+05$ & 3.4 & 60 & 85 & 1302 \\
\hline Individual 24 & 2134 & 2517 & 21.2 & $6.1 \mathrm{E}+05$ & 2.9 & 65 & 92 & 1302 \\
\hline Individual 25 & 2145 & 2189 & 18.2 & $5.2 \mathrm{E}+05$ & 2.1 & 75 & 108 & 1302 \\
\hline Individual 26 & 2174 & 1613 & 13.6 & $3.9 \mathrm{E}+05$ & 1.6 & 97 & 139 & 1302 \\
\hline Individual 27 & 2191 & 1471 & 12.4 & $3.5 \mathrm{E}+05$ & 1.6 & 106 & 152 & 1302 \\
\hline Individual 28 & 2163 & 1780 & 15.1 & $4.3 \mathrm{E}+05$ & 1.9 & 87 & 125 & 1302 \\
\hline Individual 29 & 2136 & 2438 & 21.3 & $6.1 \mathrm{E}+05$ & 3.9 & 62 & 88 & 1302 \\
\hline Individual 30 & 2132 & 2586 & 21.8 & $6.2 \mathrm{E}+05$ & 3.0 & 63 & 90 & 1302 \\
\hline Individual 31 & 2158 & 1874 & 16.0 & $4.6 \mathrm{E}+05$ & 2.2 & 82 & 118 & 1302 \\
\hline Individual 32 & 2135 & 2480 & 20.9 & $6.0 \mathrm{E}+05$ & 2.8 & 66 & 94 & 1302 \\
\hline Individual 33 & 2187 & 1495 & 12.6 & $3.6 \mathrm{E}+05$ & 1.7 & 104 & 149 & 1302 \\
\hline Individual 34 & 2130 & 2690 & 22.8 & $6.5 \mathrm{E}+05$ & 3.3 & 60 & 86 & 1302 \\
\hline Individual 35 & 2146 & 2142 & 18.5 & $5.3 \mathrm{E}+05$ & 2.9 & 71 & 101 & 1302 \\
\hline
\end{tabular}

\section{Conclusions}

The application of the $\mathrm{CoDeO}$ methodology to the case study application resulted in significant saving in material and cost. The holistic approach over manufacturing optimization enable the creation of a reliable model for optimizing both economic and technological sides of a NNS manufacturing chain.

$\mathrm{CoDeO}$ can be mathematically integrated with other models for generating a holistic production models and concurrent optimization.
Similarly to other general models, the methodology is not suitable for non-expert users, because of the choices that need to be made during the models development and algorithm setting phases. Variable and targets definition are critical for the optimization, however, in the context of a global NNS process chain, the general model developed defines the key outcomes (i.e. objective functions definition) and limit possible search space (i.e. variable selection).Further details of the methodology can be found in Marini (2020). 
Table 7 Current and optimized (Individual 20) geometry, process parameters and results (optimization objectives and constraints)

\begin{tabular}{lll}
\hline $\begin{array}{l}\text { Variables, constraints and optimiza- } \\
\text { tion objectives }\end{array}$ & GA optimized & NNS process \\
\hline Geometry & & \\
OD [mm] & 495 & 520 \\
ID [mm] & 410 & 400 \\
$O D_{b}[\mathrm{~mm}]$ & 478 & 492 \\
$l_{f}[\mathrm{~mm}]$ & 65 & 55 \\
$L_{f}[\mathrm{~mm}]$ & 415 & 420 \\
Process parameters & & \\
a [mm] & 7.1 & 3.4 \\
$\mathrm{n}$ [RPM] & 254 & 100 \\
F [mm/1] & 0.25 & 0.23 \\
Optimized objectives and constraints & & \\
Cutting force [N] & 2640 & 1650 \\
Cutting power [kW] & 22.3 & 4.8 \\
Machining removal rate [mm $\left.{ }^{3} / \mathrm{min}\right]$ & $6.4 \mathrm{E}+05$ & $3.9 \mathrm{E}+05$ \\
Surface roughness [Ra] & 3.2 & 6.4 \\
Machining time [min] & 62 & 107 \\
Material waste [kg] & 116 & 93 \\
\hline
\end{tabular}

Acknowledgements The authors want to thank WARC (Weir Advanced Research Centre) and Weir Group PLC of Glasgow for the fundamental and continuous support in this research.
Open Access This article is licensed under a Creative Commons Attribution 4.0 International License, which permits use, sharing, adaptation, distribution and reproduction in any medium or format, as long as you give appropriate credit to the original author(s) and the source, provide a link to the Creative Commons licence, and indicate if changes were made. The images or other third party material in this article are included in the article's Creative Commons licence, unless indicated otherwise in a credit line to the material. If material is not included in the article's Creative Commons licence and your intended use is not permitted by statutory regulation or exceeds the permitted use, you will need to obtain permission directly from the copyright holder. To view a copy of this licence, visit http://creativecommons.org/licenses/by/4.0/.

\section{Appendix A: Helix-turning formulation}

This appendix details the representation of the helicoidal turning ar developed to support the optimization step.

In Figs. 6 and 7, the turning helix has been displayed. In point $\mathrm{O}$, the global frame of the component is composed by the axis $x, y, z$ and the unit vectors $(\underline{i}, j, \underline{k})$. T is the point of contact of the tool. The local frame is composed of three unit vectors: $c$, tangent to the circumference, to which corresponds the cutting speed vector $v_{c} ; t$, tangent to the helix trajectory, to which corresponds the helix speed vector $v_{H} ; a$, parallel to the workpiece axis and coincident to the tool motion axis (liner trajectory), to which corresponds the feed speed vector $v_{H}$.

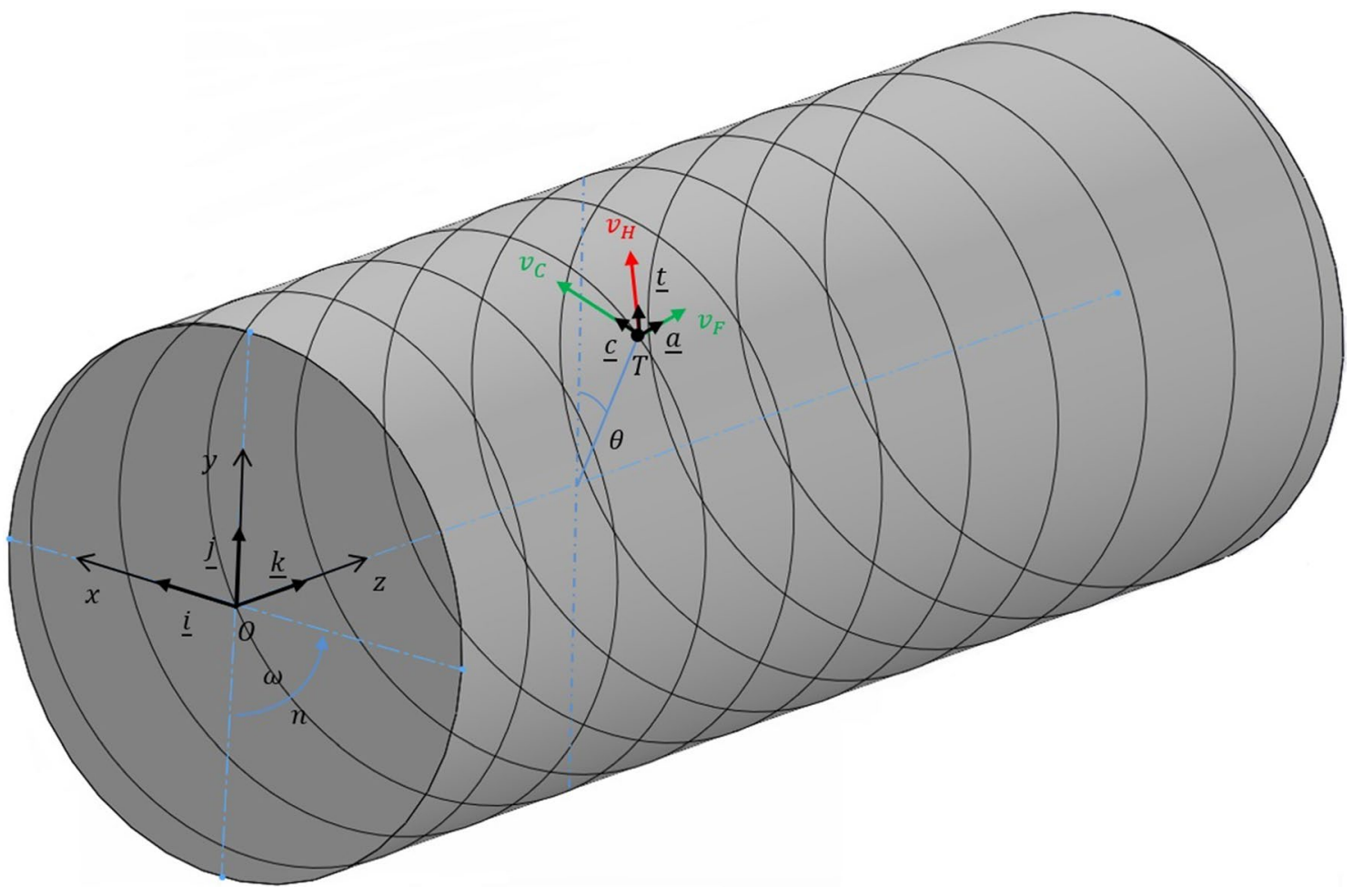

Fig. 6 Turning helix model (isometric view) (Color figure online) 


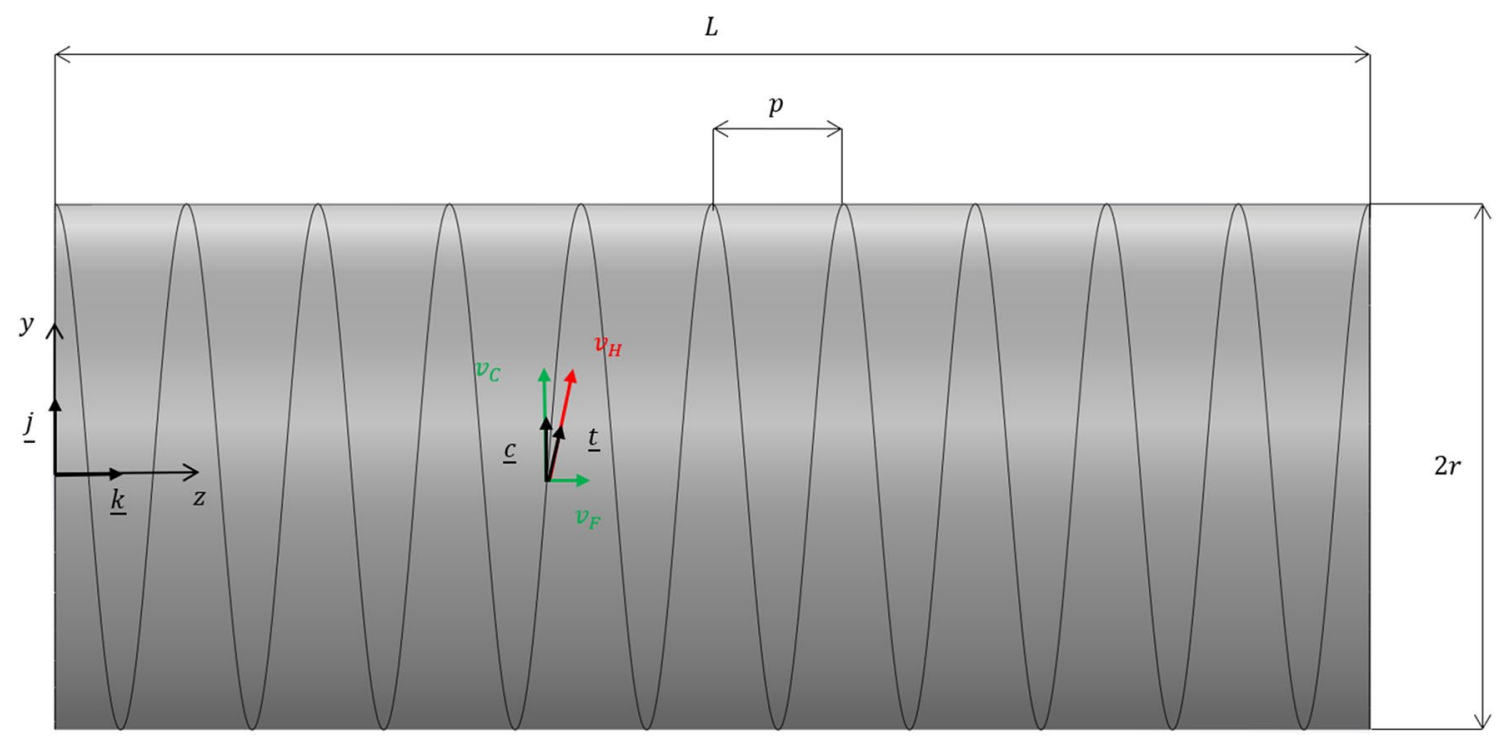

Fig. 7 Turning helix model (lateral view) (Color figure online)

The helix equation parametric equation can be expressed as in (15)

$\left\{\begin{array}{l}x=r \cos (\theta) \\ y=r \sin (t \theta) \\ z=c \theta\end{array}\right.$

With, $\theta \in[0, k \pi] ; x, y \in[0, r] ; z \in[0, L]$.

The main features of the helix are: $c=\frac{p}{2 \pi}$; Helix pitch, $p$; Slope, $\frac{c}{p}$; Number of turns, $\frac{\theta_{T}}{2 \pi}$; Torsion, $\tau=\frac{c}{r^{2}+c^{2}}$; Curvature, $k=\frac{r}{r^{2}+c^{2}}$.

The velocity of a point on the helix can be written as (16)

$$
\begin{aligned}
\underline{v} & =v_{x} \underline{i}+v_{y} \underline{j}+v_{z} \underline{k}=\frac{d x}{d t} \underline{i}+\frac{d y}{d t} \underline{j}+\frac{d z}{d t} \underline{k} \\
& =\left(-r \frac{d \theta}{d t} \sin (\theta)\right) \underline{i}+r \frac{d \theta}{d t} \cos (\theta) \underline{j}+c \frac{d \theta}{d t} \underline{k}
\end{aligned}
$$

Velocity vector can be written as in (17)

$$
\begin{aligned}
\underline{v} & =\|v\| \underline{t} \\
& =\sqrt{v_{x}^{2}+v_{y}^{2}+v_{z}^{2}} \underline{t}=\sqrt{\frac{d^{2} \theta}{d t^{2}}\left(r^{2}\left(\sin ^{2}(\theta)+\cos ^{2}(\theta)\right)+c^{2}\right) \underline{t}} \\
& =\sqrt{\left(r^{2}+c^{2}\right)} \frac{d \theta}{d t} t
\end{aligned}
$$

If the motion is uniform, it is possible to write (18)

$$
\operatorname{fracd}^{2} \theta d t^{2}=0, \frac{d \theta}{d t}=\omega
$$

Arc Length: the total helix arc length can be derived integrating the velocity (17) as in (19) and (20)

$$
\begin{aligned}
& d s=v d t, \int_{0}^{l} d s=\int_{t_{0}}^{t}|v| d t \\
& l_{H}=\int_{t_{0}}^{t} \sqrt{\left(r^{2}+c^{2}\right)} \frac{d \theta}{d t} d t=\sqrt{\left(r^{2}+c^{2}\right)} \theta_{t_{0}}^{t}
\end{aligned}
$$

Classic turning formulation The classic formulation use the following inputs:

- $F$, feed rate (mm/lap)

- $n$, spindle speed (lap/min)

- $a$, depth of cut (mm)

- $l$, working engagement $(\mathrm{mm})$

The feed velocity (21), cutting speed (22) and cutting time (23) classic formulations can be written as follows

$v_{f}=F n$

$v_{c}=\pi D_{c} n$

$t_{c}=\frac{l}{F n}$

Helix equivalence For the helix the speed can be written as (24)

$v_{H}=l_{H} / t_{c}$ 
Having the total number of turns $\theta_{T}$, the length (20) can be rewritten as (25)

$l_{H}=\sqrt{\left(r^{2}+c^{2}\right)} \theta_{T}$

Given, $z=c \theta$, with $z=L$, it is possible to write (26)

$\theta_{T}=\frac{L}{c}=\frac{L}{p / 2 \pi}$

The cutting time $t_{c}$ must be the same for both the formulations. Having $L=l$ and $t_{c}=\frac{l}{F n}$, the cutting time can be also written as (27)

$t_{c}=\frac{\sqrt{\left(r^{2}+c^{2}\right)} \frac{2 \pi L}{p}}{\sqrt{\left(r^{2}+c^{2}\right)} \omega}=\frac{2 \pi L}{p \omega}$

The hypothesis $t_{c}$ (helix) $=t_{c}$ (classic) can be dimensionally checked as in (28)

$\frac{2 \pi L}{p \omega}=\frac{L}{F n} \rightarrow\left[\frac{2 \pi L}{p \omega}\right]=\frac{m m}{\frac{m m}{r a d} \frac{r a d}{s}}=\frac{m m}{\frac{m m}{l a p} \frac{l a p}{s}}=\left[\frac{L}{F n}\right]$

Therefore, the helix pitch can be written as (29)

$p=\frac{2 \pi F n}{\omega}=60 F$

Velocity hypothesis The helix speed and the cutting and feed rate speed must be complementary, so (30) can be formulated.

$v_{H}=\sqrt{v_{c}^{2}+v_{f}^{2}}$

Hybrid formulation In (31) the helix and classic formulation are combined by substituting the $v_{H}(30)$ in cutting time equation (27), as in the (24)

$t_{c}=\frac{\sqrt{\left(r_{i}^{2}+c_{i}^{2}\right)} \frac{2 \pi L_{i}}{p_{i}}}{\sqrt{v_{c_{i}}^{2}+v_{f_{i}}^{2}}}$

With $c=\frac{p}{2 \pi}$, it is possible to write (32) and finally derive (33)

$$
\begin{aligned}
t_{c} & =\frac{\sqrt{(r)^{2}+\left(\frac{F n}{\omega}\right)^{2}} \frac{\omega L}{F n}}{\sqrt{v_{c}^{2}+v_{f}^{2}}}=\left(\frac{\frac{L^{2} \omega^{2}}{F^{2} n^{2}}\left(r^{2}+\frac{F^{2} n^{2}}{\omega^{2}}\right)}{F^{2} n^{2}+4 \pi^{2} r^{2} n^{2}}\right)^{\frac{1}{2}} \\
& =\left(\frac{L^{2}\left(\frac{\omega^{2}}{F^{2} n^{2}} r^{2}+1\right)}{\left(n^{2}(F)^{2}+4 \pi^{2} r^{2}\right)}\right)^{\frac{1}{2}}
\end{aligned}
$$

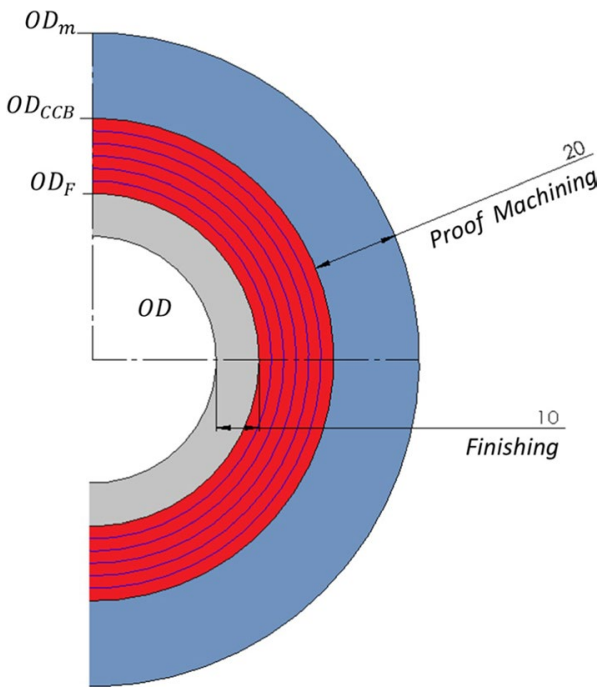

Fig. 8 External turning process scheme and nomenclature scheme (blue: proof machining zone; red: roughing zone; grey: finishing zone) (Color figure online)

$t_{c}=\frac{L}{n}\left(\frac{\frac{\omega^{2}}{F^{2} n^{2}} r^{2}+1}{F^{2}+4 \pi^{2} r^{2}}\right)^{\frac{1}{2}}$

A dimensional check (34) shows the validity of the formula (33)

$$
\begin{aligned}
t_{c} & =\left[\frac{L}{n}\left(\frac{\frac{\omega^{2}}{F^{2} n^{2}} r^{2}+1}{(F)^{2}+\pi^{2} r^{2}}\right)^{\frac{1}{2}}\right]=\frac{m m}{\frac{1}{s}}\left(\frac{\frac{\left(\frac{1}{s}\right)^{2} m m^{2}}{\frac{m m^{2}}{1} \frac{1}{s^{2}}}+1}{\left(\frac{m m}{1}\right)^{2}+m m^{2}}\right)^{\frac{1}{2}} \\
& =\frac{m m}{\frac{1}{s}}\left(\frac{1}{m m^{2}}\right)^{\frac{1}{2}}=\frac{m m}{m m / s}=s
\end{aligned}
$$

In conclusion, with $\omega=\frac{2 \pi n}{60}$ and $D=2 r$ (so $D^{2}=4 r^{2}$ ) the cutting time become (34)

$t_{c}=60 \frac{L}{n}\left(\frac{\left(\frac{\pi D}{60 F}\right)^{2}+1}{F^{2}+\pi^{2} D^{2}}\right)^{\frac{1}{2}}$

\section{Appendix B: Turning model formulation}

Referring to Fig. 8 it is possible to define: Finishing Diameters (10 mm hypothesis) 
Table 8 Turning model nomenclature

\section{Turning Model Nomenclature}

$O D$, external final diameter after finishing $(\mathrm{mm})$

$O D_{F}$, external machined diameter before finishing ( $\mathrm{mm}$ )

$O D_{C C B}$, external centrifugal casting blank diameter after proof machining $(\mathrm{mm})$ $O D_{M}$, external centrifugal casting blank diameter $(\mathrm{mm})$

$I D$, internal final diameter after finishing $(\mathrm{mm})$

$I D_{F}$, intern machined diameter before finishing $(\mathrm{mm})$

$I D_{C C B}$, internal centrifugal casting blank diameter after proof machining $(\mathrm{mm})$ $I D_{M}$, internal centrifugal casting blank diameter $(\mathrm{mm})$

$O D_{b}$, external final diameter of the bottom section after finishing ( $\mathrm{mm}$ ) $O D_{b_{F}}$, external final diameter of the bottom section before finishing $(\mathrm{mm})$ $O D_{C C B}$, external centrifugal casting blank diameter after proof machining $(\mathrm{mm})$ $O D_{M}$, external centrifugal casting blank diameter $(\mathrm{mm})$

$N_{E_{T}}$, theoretical number of turning process passes for the external zone $N_{l_{T}}$, theoretical number of turning process passes for the internal zone $N_{E b_{T}}$, theoretical number of turning process passes for the external bottom section zone $N_{E}$, rounded number of turning process passes for the external zone

$N_{I}$, rounded number of turning process passes for the internal zone

$N_{E_{b}}$, rounded number of turning process passes for the external bottom section zone $L$, component (turning) length ( $\mathrm{mm}$ ) $l$, bottom section (turning) length ( $\mathrm{mm}$ )

$a$, depth of cut (mm)

$F$, feed rate $(\mathrm{mm})$

$n$, rotational speed $(1 / \mathrm{min})$

$T_{T u}$, total turning time (min)

$t_{l N_{T}}$, total internal turning time (min)

$t_{E X_{T}}$, total external turning time (min)

$T_{E X_{b T}}$, total external bottom section turning time (min)

\section{External and Internal Turning Derivation Nomenclature}

$a_{i}$, depth of cut for the $i_{t h}$ turning operation (mm)

$F_{i}$, feed rate for the $i_{t h}$ turning operation ( $\mathrm{mm}$ )

$n_{i}$, rotational speed $(1 / \mathrm{min})$

$N$, total number of turning passes for the whole machining operation

$N_{e}$, rounded external number of turning passes

$N_{i}$, rounded internal number of turning passes

$L_{i}$, turning length for the $i_{t h}$ turning operation

(mm)

$L_{T}$, generic total turning length $(\mathrm{mm})$

$D_{i}$, total turning diameter for the $i_{t h}$ turning operation $(\mathrm{mm})$

$O D_{i}$, external turning diameter for the $i_{t h}$ turning operation (mm)

$O D_{0}$, external turning diameter for the $i_{t h}$ turning operation ( $\mathrm{mm}$ )

$O D_{T}$, external turning diameter for the $i_{t h}$ turning operation ( $\mathrm{mm}$ )

$O D_{T}$, external turning diameter for the $i_{t h}$ turning operation $(\mathrm{mm})$

$O D_{0}$, initial outer turning diameter $(\mathrm{mm})$

$O D_{f}$, final outer turning diameter ( $\left.\mathrm{mm}\right)$

$I D_{i}$, generic internal turning diameter for

the $i_{t h}$ turning operation ( $\mathrm{mm}$ )

$I D_{0}$, initial inner turning diameter $(\mathrm{mm})$

$I D_{f}$, final inner turning diameter $(\mathrm{mm})$

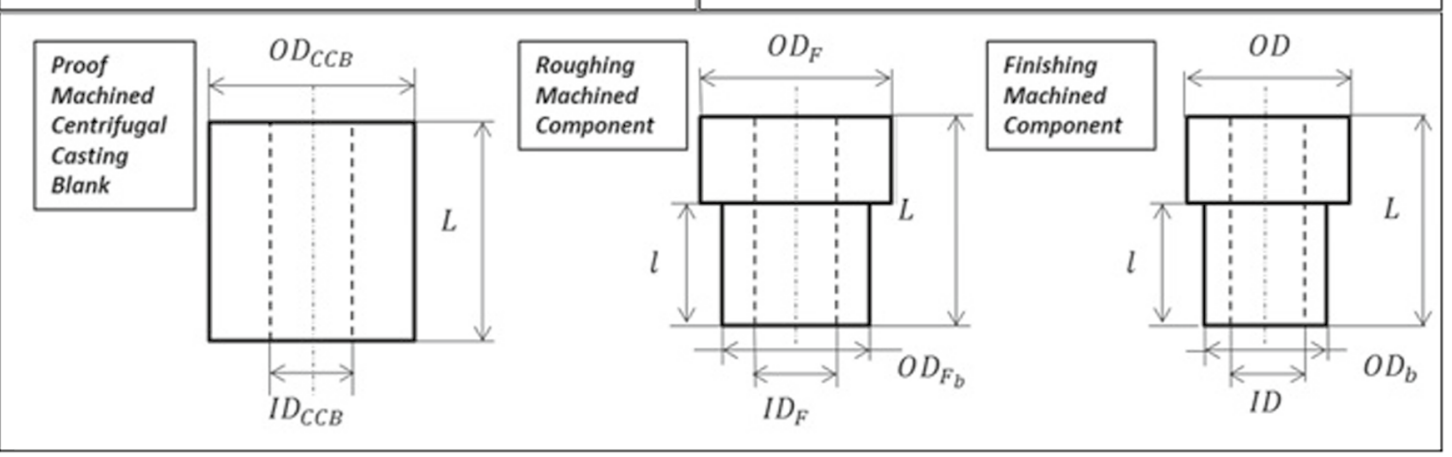


$O D_{F}=O D+10$

$O D_{F_{b}}=O D_{b}+10$

$I D_{F}=I D-10$

Roughing Machining Theoretical number of turns (Table 8; Fig. 9)

$N_{E_{T}}=\frac{O D_{C C B}-O D_{F}}{a}=\frac{O D_{m}-O D-30}{a}$

$N_{I_{T}}=\frac{I D_{F}-I D_{C C B}}{a}=\frac{I D-I D_{m}-30}{a}$

$N_{E b_{T}}=\frac{O D_{F}-O D_{F_{b}}}{a}=\frac{O D-O D_{b}}{a}$

Rounded number of turns (natural number)

$N_{E}=\operatorname{roundup}\left(\frac{O D_{m}-O D-30}{a}\right)$

$N_{E_{b}}=\operatorname{roundup}\left(\frac{I D-I D_{m}-30}{a}\right)$

$N_{I}=\operatorname{roundup}\left(\frac{O D-O D_{b}}{a}\right)$

Proof Machined Diameters (20 mm allowances from centrifugal casting blank)

$O D_{C C B}=O D+10+N_{e} a=O D_{m}-20$

$I D_{C C B}=I D+10+N_{e} a=I D_{m}-20$

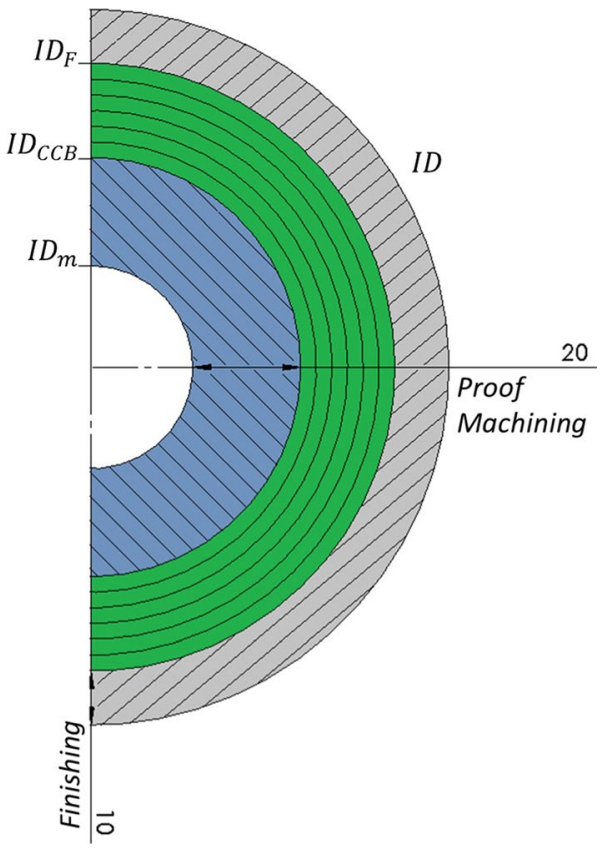

Fig. 9 External turning process scheme and nomenclature scheme (grey: finishing zone; green: roughing zone; blue: proof machining zone) (Color figure online)

- $a_{i}=$ costant $=a$

- $n_{i}=$ costant $=n$

- $F_{i}=$ constant $=F$

In addition, in all three cases, the turning length can be considered constant $\left(L_{i}=\right.$ cost $\left.=L\right)$ for a single turning operation. Therefore (47) can be written

$\sum_{i=1}^{N} L_{i}=N L_{T}$

Therefore, using the turning helix formula, the total turning time can be written as (48)

$$
\begin{gathered}
t_{T}=\sum_{i=1}^{N} t_{i}=t_{1}+t_{2}+\cdots+t_{N}=\frac{60 L}{n} \sqrt{\frac{1+\pi^{2} D_{1}^{2}}{F^{2}+\pi^{2} D_{1}^{2}}+\frac{60 L}{n_{1}} \sqrt{\frac{1+\pi^{2} D_{2}^{2}}{F^{2}+\pi^{2} D_{2}^{2}}}+\ldots} \\
\cdots+\frac{60 L}{n} \sqrt{\frac{1+\pi^{2} D_{N}^{2}}{F^{2}+\pi^{2} D_{N}^{2}}}=\frac{60 N L}{n} \sqrt{\frac{1+\pi^{2} \sum_{i=1}^{N} D_{i}}{F^{2}+\pi^{2} \sum_{i=1}^{N} D_{i}}}
\end{gathered}
$$

For a single turning operation, the machining parameters can be considered as constant, so the following hypotheses result generally valid for the turning process parameters.
Distinction needs to be made for the external and internal turning.

$$
\text { In external turning case }\left(D_{i}=O D_{i}\right)
$$


$O D_{i}=O D_{0}-\left(a_{1}+a_{2}+\cdots+a_{i}\right)$

Although with $a=$ constant it becomes

$O D_{i}=O D_{0}-\left(a+2 a+3 a+\cdots+N_{i} a\right)=O D_{0}-\sum_{i=1}^{N_{i_{E}}} i(a)$

The last sum can be written as

$\sum_{i=1}^{N_{e}} i(a)=\frac{N_{e}\left(N_{e}+1\right) a}{2}$

Therefore

$O D_{i}=O D_{0}-\frac{N_{e}\left(N_{e}+1\right) a}{2}$

Therefore

$\sum_{i=1}^{N_{e}} O D_{i}=N_{e} O D_{0}-\frac{N_{e}\left(N_{i_{e}}+1\right) a}{2}$

Similar conclusions can be drafted for the internal turning case $\left(D_{i}=\left(I D_{i}\right)\right)$ The general inner diameter after $i$-passes can be written as

$I D_{i}=I D_{0}+\left(a_{1}+a_{2}+\cdots+a_{i}\right)$

Therefore, dually to the outer diameter case

$I D_{i}=I D_{0}+\frac{N_{i}\left(N_{i}+1\right) a}{2}$

Therefore the total external turning time formula become

$t_{E_{T}}=\frac{60 N_{e} L_{T}}{n} \sqrt{\frac{1+\pi^{2}\left(N_{e} O D_{0}-\frac{N_{e}\left(N_{e}+1\right) a}{2}\right)^{2}}{F^{2}+\pi^{2}\left(N_{e} O D_{0}-\frac{N_{e}\left(N_{e}+1\right) a}{2}\right)^{2}}}$

With $N_{e}=\operatorname{round}\left(\frac{O D_{0}-O D_{f}}{a}\right)$

Similarly the total internal turning time

$t_{I_{T}}=\frac{60 N_{i} L}{n} \sqrt{\frac{1+\pi^{2}\left(N_{i} I D_{0}+\frac{N_{i}\left(N_{i}+1\right) a}{2}\right)^{2}}{F^{2}+\pi^{2}\left(N_{I} I D+\frac{N_{i}\left(N_{i}+1\right) a}{2}\right)^{2}}}$

In the examined case, the total turning time can be written as

$$
\begin{aligned}
& T_{T u}=t_{I N_{T}}+t_{E X_{T}}+t_{E X_{b T}} \\
&=\frac{60 N_{E} L}{n} \sqrt{\frac{1+\pi^{2}\left(N_{E}\left((O D-10)-\frac{N_{E}\left(N_{i_{E}}+1\right) a}{2}\right)\right)^{2}}{F^{2}+\pi^{2}\left(N_{E}\left((O D-10)-\frac{N_{i_{E}}\left(N_{i_{E}}+1\right) a}{2}\right)\right)^{2}}} \\
&+\frac{60 N_{I} L}{n} \sqrt{\frac{1+\pi^{2}\left(N_{I}\left((I D+10)+\frac{N_{I}\left(N_{I}+1\right) a}{2}\right)\right)^{2}}{F^{2}+\pi^{2}\left(N_{I}\left((I D+10)+\frac{N_{I}\left(N_{I}+1\right) a}{2}\right)\right)^{2}}} \\
&+\frac{60 N_{E_{b}} l}{n_{i}} \sqrt{\frac{1+\pi^{2}\left(N_{E_{b}}\left(\left(O D_{b}-10\right)-\frac{N_{E_{b}}\left(N_{E_{b}}+1\right) a}{2}\right)\right)^{2}}{F_{i}^{2}+\pi^{2}\left(N_{E_{b}}\left(\left(O D_{b}-10\right)-\frac{N_{E_{b}}\left(N_{E_{b}}+1\right) a}{2}\right)\right)^{2}}}
\end{aligned}
$$

\section{References}

Al-Ansary, M. D., \& Deiab, I. M. (1997). Concurrent optimization of design and machining tolerances using the genetic algorithms method. International Journal of Machine Tools and Manufacture, 37(12), 1721-1731.

Alberti, N., \& Perrone, G. (1999). Multipass machining optimization by using fuzzy possibilistic programming and genetic algorithms. Proceedings of the Institution of Mechanical Engineers, Part B: Journal of Engineering Manufacture, 213, 261-273.

Altan, T., \& Allen, Miller R. (1990). Design for forming and other near net shape manufacturing processes. CIRP Annals - Manufacturing Technology, 39(2), 609-620.

Amiolemhen, P. E., \& Ibhadode, A. O. A. (2004). Application of genetic algorithms: Determination of the optimal machining parameters in the conversion of a cylindrical bar stock into a continuous finished profile. International Journal of Machine Tools and Manufacture, 44(12-13), 1403-1412.

Aydoğan, E., Delice, Y., Özcan, U., Gencer, C., \& Bali, Ö. (2019). Balancing stochastic u-lines using particle swarm optimization. Journal of Intelligent Manufacturing, 30(1), 97-111.

Bäck, T., \& Schwefel, H. P. (1993). An overview of evolutionary algorithms for parameter optimization. Evolutionary Computation, $1(1), 1-23$.

Bi, Z. M., \& Zhang, W. J. (2001). Concurrent optimal design of modular robotic configuration. Journal of Robotic systems, 18(2), $77-87$.

Burnell, L. J., Priest, J. W., \& Briggs, K. (1991). An intelligent decision theoretic approach to producibility optimization in conceptual design. Journal of Intelligent Manufacturing, 2(3), 189-196.

Caporalli, A., Gileno, L. A., \& Button, S. T. (1998). Expert system for hot forging design. Journal of Materials Processing Technology, 80-81, 131-135.

Cus, F., \& Balic, J. (2003). Optimization of cutting process by GA approach. Robotics and Computer-Integrated Manufacturing, 19(1-2), 113-121. 
D’Addona, D. M., \& Teti, R. (2013). Genetic algorithm-based optimization of cutting parameters in turning processes. Procedia CIRP, 7, 323-328.

Davidson, M. J., Balasubramanian, K., \& Tagore, G. R. N. (2008). Experimental investigation on flow-forming of AA6061 alloy: A Taguchi approach. Journal of Materials Processing Technology, 200(1-3), 283-287.

Deb, K. (2001). Multi-objective optimization using evolutionary algorithm. London: Wiley.

Del Prete, A., Franchi, R., Cacace, S., \& Semeraro, Q. (2019). Optimization of cutting conditions using an evolutive online procedure. Journal of Intelligent Manufacturing, 1-19.

Denkena, B., Behrens, B. A., Charlin, F., \& Dannenberg, M. (2011). Integrative process chain optimization using a genetic algorithm. Production Engineering, 6(1), 29-37.

Fleury, G., Goujon, J. Y., Gourgand, M., \& Lacomme, P. (1999). Multi-agent approach and stochastic optimization: Random events in manufacturing systems. Journal of intelligent Manufacturing, 10(1), 81-101.

Fonseca, C. M., \& Fleming, P. J. (1995). An overview of evolutionary algorithms in multiobjective optimization. Evolutionary Computation, 3(1), 1-16.

Fonseca, C. M., \& Fleming, P. J. (1998). Multiobjective optimization and multiple constraint handling with evolutionary algorithms. I. A unified formulation. IEEE Transactions on Systems, Man, and Cybernetics-Part A: Systems and Humans, 28(1), 26-37.

Giachetti, R. E. (1998). A decision support system for material and manufacturing process selection. Journal of Intelligent Manufacturing, 9(3), 265-276.

Gupta, K. (2019). Near net shape manufacturing processes. Berlin: Springer.

Ha, A. Y., \& Porteus, E. L. (1995). Optimal timing of reviews in concurrent design for manufacturability. Management Science, 41(9), 1431-1447.

Huang, M. F., Zhong, Y. R., \& Xu, Z. G. (2005). Concurrent process tolerance design based on minimum product manufacturing cost and quality loss. The International Journal of Advanced Manufacturing Technology, 25(7-8), 714-722.

Kalpakjian, S., \& Schimd, S. R. (2009). Manufacturing engineering and technology (6th ed.). Upper Saddle River: Prentice Hall.

Khan, Z., Prasad, B., \& Singh, T. (1997). Machining condition optimization by genetic algorithms and simulated annealing. Computers \& Operations Research, 24(7), 647-657.

Lee, S. S., \& Wang, H. P. B. (1992). Modified simulated annealing for multiple-objective engineering design optimization. Journal of Intelligent Manufacturing, 3(2), 101-108.

Li, Q., Zhang, W. J., \& Chen, L. (2001). Design for control-a concurrent engineering approach for mechatronic systems design. IEEE/ASME Transactions on Mechatronics, 6(2), 161-169.

Liu, H., Wang, Y., Tu, L., Ding, G., \& Hu, Y. (2019). A modified particle swarm optimization for large-scale numerical optimizations and engineering design problems. Journal of Intelligent Manufacturing, 30(6), 2407-2433.

Liu, Z., Li, X., Wu, D., Qian, Z., Feng, P., \& Rong, Y. (2019). The development of a hybrid firefly algorithm for multi-pass grinding process optimization. Journal of Intelligent Manufacturing, 30(6), 2457-2472.

Marini, D., \& Corney, J. R. (2017). A methodology for near net shape process feasibility assessment. Production \& Manufacturing Research, 5(1), 390-409.

Marini D., Cunningham D., \& Corney J. R. (2017). Near net shape manufacturing of metal: A review of approaches and their evolutions. Proceedings of the Institution of Mechanical Engineers, Part B: Journal of Engineering Manufacture.

Marini D. (2020). An application protocol for near net shape technologies implementation. Ph.D. Thesis, University of Strathclyde.
Mukherjee, I., \& Ray, P. K. (2006). A review of optimization techniques in metal cutting processes. Computers and Industrial Engineering, 50(1-2), 15-34.

Onwubolu, G. C., \& Kumalo, T. (2001). Optimization of multipass turning operations with genetic algorithms. International Journal of Production Research, 39(16), 3727-3745.

Ouyang, P. R., Li, Q., Zhang, W. J., \& Guo, L. S. (2004). Design, modeling and control of a hybrid machine system. Mechatronics, 14(10), 1197-1217.

Pierreval, H., \& Tautou, L. (1997). Using evolutionary algorithms and simulation for the optimization of manufacturing systems. IIE Transactions, 29(3), 181-189.

Ramesh, R., Jerald, J., Page, T., \& Arunachalam, S. (2009). Concurrent tolerance allocation using an artificial neural network and continuous ant colony optimisation. International Journal of Design Engineering, 2(1), 1-25.

Rampersad, H. K. (1995). Concurrent design of product, process and robotic assembly system. Assembly Automation, 15(1), 21-28.

Rao, R. V., Rai, D. P., \& Balic, J. (2019). Multi-objective optimization of abrasive waterjet machining process using jaya algorithm and promethee method. Journal of Intelligent Manufacturing, 30(5), 2101-2127.

Rao, R. V., Rai, D Pd, \& Balic, J. (2018). Multi-objective optimization of machining and micro-machining processes using non-dominated sorting teaching-learning-based optimization algorithm. Journal of Intelligent Manufacturing, 29(8), 1715-1737.

Saif, U., Guan, Z., Zhang, L., Zhang, F., Wang, B., \& Mirza, J. (2019). Multi-objective artificial bee colony algorithm for order oriented simultaneous sequencing and balancing of multi-mixed model assembly line. Journal of Intelligent Manufacturing, 30(3), $1195-1220$

Saravanan, R., Asokan, P., \& Vijayakumar, K. (2003). Machining parameters optimisation for turning cylindrical stock into a continuous finished profile using genetic algorithm $(\mathrm{GA})$ and simulated annealing (SA). International Journal of Advanced Manufacturing Technology, 21(1), 1-9.

Sardinas, R. Q., Santana, M. R., \& Brindis, E. A. (2006). Genetic algorithm-based multi-objective optimization of cutting parameters in turning processes. Engineering Applications of Artificial Intelligence, 19(2), 127-133.

Schrader R. S. (2003). Optimization of cost and cycle time for multipass turning operation using GA, genetic algorithm at the university of Illinois fall 2002.

Srinivas, N., \& Deb, K. (1994). Muiltiobjective optimization using nondominated sorting in genetic algorithms. Evolutionary Computation, 2(3), 221-248.

The Weir Group PLC. (2015). Annual report and financial statements retrieved from Weir Group PLC.

Wang, J., Kuriyagawa, J., Wei, X. P., \& Guo, D. M. (2002). Optimization of cutting conditions for single pass turning operations using a deterministic approach. International Journal of Machine Tools and Manufacture, 42(9), 1023-1033.

Yang, S. H., \& Natarajan, S. H. (2010). Multi-objective optimization of cutting parameters in turning process using differential evolution and non-dominated sorting genetic algorithm-ii approaches. The International Journal of Advanced Manufacturing Technology, 49(5-8), 773-784.

Yildiz, A. R., \& Ozturk, F. (2006). Hybrid enhanced genetic algorithm to select optimal machining parameters in turning operation. Proceedings of the Institution of Mechanical Engineers, Part B: Journal of Engineering Manufacture, 220, 2041-2053.

Yildiz, A. R. (2013). Hybrid Taguchi-differential evolution algorithm for optimization of multi-pass turning operations. Applied Soft Computing, 13(3), 1433-1439. 
Minghai, Y., Hongyan, Y., Jinting, H., \& Aimin, J. (2019). Reconfigurable assembly line balancing for cloud manufacturing. Journal of Intelligent Manufacturing, 30(6), 2391-2405.

Zainal, N., Zain, A. M., Radzi, N. H. M., \& Othman, M. R. (2016). Glowworm swarm optimization (GSO) for optimization of machining parameters. Journal of Intelligent Manufacturing, 27(4), 797-804.

Zhang, W. J., \& Wang, J. W. (2016). Design theory and methodology for enterprise systems. Enterprise Information Systems, 10(3), $245-248$.
Zhang, W. J., Wang, J. W., \& Lin, Y. (2019). Integrated design and operation management for enterprise systems. Enterprise Information Systems, 13(4), 424-429.

Zitzler, E., Deb, K., \& Thiele, L. (2000). Comparison of multiobjective evolutionary algorithms: Empirical results. Evolutionary Computation, 8(2), 173-195.

Publisher's Note Springer Nature remains neutral with regard to jurisdictional claims in published maps and institutional affiliations. 